This is a post-print of the following article: Maresca, F.; Kouznetsova, V.G.; Geers, M.G.D.; Curtin, W.A. Acta Materialia 2018,, 463-478.. The formal publication is available at http://dx.doi.org/10.1016/j.actamat.2018.06.028 (C) 2018. This manuscript version is made available under the CC-BY-NC-ND 4.0 license http://creativecommons.org/licenses/by-nc-nd/4.0/

\title{
Contribution of austenite-martensite transformation to deformability of advanced high strength steels: from atomistic mechanisms to microstructural response
}

\author{
F. Maresca ${ }^{\mathrm{a}, *}$, V.G. Kouznetsova ${ }^{\mathrm{b}}$, M.G.D. Geers ${ }^{\mathrm{b}}$, W.A. Curtin ${ }^{\mathrm{a}}$ \\ ${ }^{a}$ Institute of Mechanical Engineering, École Polytechnique Fédérale de Lausanne, Lausanne, Switzerland \\ ${ }^{b}$ Department of Mechanical Engineering, Eindhoven University of Technology, \\ P.O. Box 513, 5600 MB Eindhoven, The Netherlands
}

\begin{abstract}
Steels combining austenite (fcc) with lath martensite (bcc) in nanolaminate microstructures are tough, resistant to hydrogen-embrittlement, and inexpensive, making them attractive for many technological applications. Austenite provides plastic deformation while martensite provides strength, but the nanoscale processes that control plasticity in the austenite layers are not fully established. Recent atomistic simulations and crystallographic theory reveal a unified understanding of the structure and motion of the fcc austenite-bcc (lath) martensite interface in steels, with transformation strains up to $\sim 90 \%$ in Fe-C alloys. In this paper, the atomistic behavior is connected to the ductility of nanolaminate microstructures. First, the mechanical response of the atomistic fcc/bcc interface under shear loading is analyzed. The interface motion follows a Schmid-type law for resolved shear stresses in the transformation direction. Furthermore, the forward fcc-to-bcc transformation is spontaneous while the reverse bcc-to-fcc transformation requires high stress. The asymmetry correlates well with the Peierls stresses for fcc and bcc screw dislocations, respectively. Second, the atomistic results guide the formulation of a two-scale continuum model for the phase transformation. The multi-scale strategy adopted here accounts for the relevant nano-scale mechanisms and enables modeling the mechanical response of real martensite microstructures, up to the scale of tens of micrometers - which would be untractable with direct atomistic simulations. Multi-scale simulations show that forward transformation contributes significantly to the apparent plasticity in lath martensite. This reinforces recent work highlighting the importance of such nanoscale austenite films for achiev-
\end{abstract}

\footnotetext{
${ }^{*}$ Corresponding author. E-mail: francesco.maresca@epfl.ch
} 
This is a post-print of the following article: Maresca, F.; Kouznetsova, V.G.; Geers, M.G.D.; Curtin, W.A. Acta Materialia 2018,, 463-478. The formal publication is available at http://dx.doi.org/10.1016/j.actamat.2018.06.028 @ 2018. This manuscript version is made available under the CC-BY-NC-ND 4.0 license http://creativecommons.org/licenses/by-nc-nd/4.0/

ing ductility and toughness in lath martensite. Overall, the present work demonstrates how atomistic insights can directly inform continuum models of microstructural deformation, and points toward directions for material control and optimization to achieve enhanced mechanical performance in these steels.

Keywords: fcc/bcc interface; TRIP-maraging; Quenched and Partitioned; Nanolaminate austenite-martensite steels; Lath martensite

\section{Introduction}

Multi-phase steels characterized by fcc austenite/bcc martensite nanolaminate microstructure (e.g. quenched and partitioned Q\&P [1], TRIP-Maraging steels [2, 3], AHSS with chemical patterning of austenite [4]) are currently the subject of intense research efforts because of their high toughness and resistance to hydrogen-embrittlement [5]. Due to their technological relevance, it is necessary to understand the physical mechanisms responsible for plasticity and damage behaviour at small scales, which in turn determine the macroscopic performance. A key issue is the effect of the austenite phase on the mechanical behaviour, especially when it is only a few nanometers thick (such as in low $\mathrm{C}$ lath martensite [6]). Due to the very small volume fractions, the thin austenite films are often neglected. Experiments show that, in low C martensite, localized plasticity occurs at lath boundaries [7, 8]. Recent theory [9-11] shows that such localized plasticity can be explained by the presence of thin austenite films retained at lath boundaries, provided they can plastically deform. However, the nanoscale process controlling plasticity of such thin layers remains unclear.

Recently, an atomistic study of the fcc austenite-bcc lath martensite interface was able to reproduce all the main experimental features [12], especially the interface defect structure, its motion, and the resulting transformation strain. This motivated a new crystallographic theory of fcc/bcc martensitic transformations. The theory predicts that the transformation strain depends on the fcc/bcc lattice parameter ratio, and can reach $\sim 94 \%$ shear strain, more than three times the commonly accepted value ( 30\%, which was not accurately established [13], as pointed out in [14]). Such a contribution to deformation is well above any previous expectations [15]. Furthermore, deformation due to transformation in nanoscale austenite films could contribute to significant apparent plastic straining at nanoscale sizes where dislocation plasticity requires high stresses. Motivated by these promising aspects, in this work we examine the 
This is a post-print of the following article: Maresca, F.; Kouznetsova, V.G.; Geers, M.G.D.; Curtin, W.A. Acta Materialia 2018,, 463-478.. The formal publication is available at http://dx.doi.org/10.1016/j.actamat.2018.06.028 @ 2018. This manuscript version is made available under the CC-BY-NC-ND 4.0 license http://creativecommons.org/licenses/by-nc-nd/4.0/

atomistic transformation process under shear in more detail, develop a continuum-level model for the transformation plasticity, and demonstrate the contributions of nanoscale austenite transformation to the overall plastic deformation in realistic lath microstructures.

Specifically, we have performed atomistic simulations of shear deformation parallel to the atomistic fcc-bcc interface of Ref. [12] and clarified the dependence of interface motion (and hence phase transformation) on the loading direction. The transformation follows a Schmidtype behaviour, i.e. it is activated at a critical resolved shear stress along the phase transformation plane and direction. The interface defect structure and higher energy of the fcc phase relative to bcc ensure that the reverse transformation (bcc to fcc) is not spontaneous. Deformation along the direction transverse to transformation is accommodated by elastic deformation up to high stresses. Altogether, interface propagation can be described by a crystal plasticity-type slip law. The deformation of the martensite/austenite aggregate is then incorporated in a standard continuum mechanics framework for transformation-induced plasticity (TRIP) analogous to [16]. Following the multi-scale approach for the modeling of nanolaminate austenite-martensite microstructures developed by Maresca et al. [10] and using the new phase transformation model, we show that phase transformation is a feasible nanoscale mechanism for the observed plastic behaviour and stress-strain response of real martensitic microstructures [7]. The new model can be used for the simulation of polycrystals where austenite transforms, providing new potential for design of steels characterized by nanolaminate austenite-martensite microstructures $[1-5]$.

The first novel contribution of this paper, compared to previous work $[10,12,16]$, is an understanding of the controlling mechanism for the stress dependence of the transformation. The Schmid-type activation of the interface and the origin of the asymmetry in forward and reverse transformation are demonstrated here for the first time. The second novel contribution of this paper is the demonstration that nanolaminate austenite can provide an essential contribution to the overall plasticity of martensitic microstructures through phase transformation.

The paper is structured as follows. Section 2 analyzes the mechanical response of the fcc/bcc interface atomistic model of [12], which clarified the crystallography of the fcc-bcc transformation but did not address what triggers the transformation mediated by interface glide. Section 3 shows, via multi-scale simulations, that fcc to bcc transformation can contribute to the exper- 
This is a post-print of the following article: Maresca, F.; Kouznetsova, V.G.; Geers, M.G.D.; Curtin, W.A. Acta Materialia 2018,, 463-478.. The formal publication is available at http://dx.doi.org/10.1016/j.actamat.2018.06.028 (C) 2018. This manuscript version is made available under the CC-BY-NC-ND 4.0 license http://creativecommons.org/licenses/by-nc-nd/4.0/

imentally observed plasticity of lath martensite microstructures. To model this phenomenon, we have first simplified the general framework presented in [16] and then adapted it to the case of the crystallography of lath martensite. This results in a nanolaminate model having the same structure as [10], but where inelastic deformation is due to phase transformation rather than dislocation-mediated plasticity. This whole body of new multi-scale work on nanolaminate austenite-martensite microstructures forms a basis for Section 4, which presents a detailed discussion of how the present findings contribute to understanding multiple unsolved aspects in the current literature on martensitic steels and austenite to martensite phase transformation in steels. The paper ends with conclusions summarizing the main results of this work.

\section{Mechanical behaviour of the fcc/bcc interface}

We consider a fully periodic bcc-fcc bicrystal, thoroughly described and analyzed previously in [12]. The bicrystal has an fcc-bcc interface characterized by the misorientation $\theta=11^{\circ}$ between crystallographic $(111)_{\text {fcc }} \|(011)_{\text {bcc }}$ plane and interface orientation (habit plane), which induces steps along $[\overline{1} 01]_{\mathrm{fcc}}$, and by the angle $\varphi=4.75^{\circ}$ between $[\overline{1} 01]_{\mathrm{fcc}}$ and $[1 \overline{1} 1]_{\mathrm{bcc}}$ around $[111]_{\mathrm{fcc}}$, defining the orientation relationship. The angles $(\theta, \varphi)$ are within the range of experimental observations. Atomistic simulations are performed with the LAMMPS package [17] using the MEAM-T interatomic potential developed in [18], which captures the essential properties of fcc and bcc phases for pure Fe. Details on the interatomic potential and the method for constructing the interface are provided in [12]. We note that the $\mathrm{T}=0 \mathrm{~K}$ fcc-bcc energy difference predicted by this potential is $\sim 10 \mathrm{meV} /$ atom, and that the fcc phase is metastable (namely a local minimum) with respect to bcc [18]. Both features enable construction of a metastable fcc-bcc interface and analysis of its structure and motion, which would be unfeasible if the energy difference between the two phases would be too large, or if the fcc phase would not be a local energy minimum. Figure 1a shows the bicrystal viewed along $[\overline{1} 01]_{\mathrm{fcc}}$. Figure 1b shows a typical interface ledge and reveals the important interface defects as $\frac{a_{\mathrm{fcc}}}{2}[\overline{101}]$ screw dislocations gliding on $(111)_{\mathrm{fcc}}$ and $\frac{a_{\mathrm{bcc}}}{2}[1 \overline{1} 1]$ screw dislocations with kinks gliding on $(\overline{101})_{\mathrm{bcc}}$.

Here we focus on the mechanical response of the interface under applied shear loading along different directions. We apply in-plane shear deformation along various angles $\alpha \in\left[-180^{\circ}, 180^{\circ}\right]$ where the angle $\alpha$ is measured on the interface $(232)_{\text {fcc }}$ plane starting from $[101]_{\text {fcc }}$ in the clockwise direction, see inset in Figure 2. Appendix A provides details about the method used 
This is a post-print of the following article: Maresca, F.; Kouznetsova, V.G.; Geers, M.G.D.; Curtin, W.A. Acta Materialia 2018,, 463-478. The formal publication is available at http://dx.doi.org/10.1016/j.actamat.2018.06.028 @ 2018. This manuscript version is made available under the CC-BY-NC-ND 4.0 license http://creativecommons.org/licenses/by-nc-nd/4.0/

for the application of the shear load on the computational cell. For each loading direction, the components of the Cauchy stress tensor $\boldsymbol{\Sigma}$ of the simulation cell are computed [17]. The resolved shear stress $\tau$ is calculated as

$$
\tau=\Sigma:\left(\mathbf{m} \otimes \mathbf{n}^{(1)}\right)
$$

where $\mathbf{m}$ is the vector aligned with the loading direction and $\mathbf{n}^{(1)}$ is the interface normal. In Eq. (1) $\mathbf{A}: \mathbf{B}=A_{i j} B_{i j}$ with Einstein summation over the repeated indices. The symbol $\otimes$ indicates a tensor (or dyadic) product between two vectors. Simulations are performed at $\mathrm{T}=0 \mathrm{~K}$; simulations at finite- $\mathrm{T}$ would be limited to short times and unrealistic strain rates, and thus providing little or no additional useful quantitative information, as it will become clear from the discussion below. As shown in Figure 1 and detailed in [12], the interface structure is composed of interface dislocations that carry the transformation by gliding. This does not require thermal activation since these defects are not nucleated but constitute the already existing interface structure. Thus, $\mathrm{T}=0 \mathrm{~K}$ simulations accurately reveal the important phenomena. Implications for finite-temperature behaviour are addressed in the Discussion section.

At a critical resolved shear stress $\tau_{c}$, the interface moves carrying the transformation from/to fcc to/from bcc. Figure 2 shows $\tau$ vs the applied displacement of the bicrystal for loads applied along the transformation direction $\left(\alpha_{\mathrm{tr}}=19.5^{\circ}[12]\right)$ and along the transverse direction $\alpha_{\perp}=$ $-70.5^{\circ}$. Along $\alpha_{\mathrm{tr}}$, a forward applied load drives the fcc to bcc transformation at less than $1 \mathrm{MPa}$. The interface propagates in a stick-slip manner, as reflected by the jagged curve, with negative stresses observed because the simulation is displacement-controlled while the interface moves in discrete atomic increments. The origin of the stress fluctuations is the presence of the weak pinning points that make the interface metastable, see Appendix A. The forward transformation is thus essentially spontaneous. This glissile and athermal nature of the interface in pure Fe was already shown in [12]. For loading in the opposite direction, the reverse transformation from bcc to fcc occurs, but at a much higher critical stress of $\tau_{c} \simeq 340$ MPa. For loading applied along the transverse direction $\alpha_{\perp}$, the material response is elastic up to stresses above $\sim 1.5 \mathrm{GPa}$ where dislocations are emitted into the bulk phases. There is no transformation and the material response is similar to that of a typical bimaterial interface or grain boundary [21]. 
This is a post-print of the following article: Maresca, F.; Kouznetsova, V.G.; Geers, M.G.D.; Curtin, W.A. Acta Materialia 2018,, 463-478.. The formal publication is available at http://dx.doi.org/10.1016/j.actamat.2018.06.028 @ 2018. This manuscript version is made available under the CC-BY-NC-ND 4.0 license http://creativecommons.org/licenses/by-nc-nd/4.0/

The in-plane yield locus, or critical shear stress as a function of loading direction, is shown in Figure 3. This yield surface is determined using the maximum shear stress at which a displacement of magnitude $b$ is accommodated by inelastic phenomena (either phase transformation or defect emission) for each loading direction $\alpha$. Note that, except for loads applied close to $\alpha_{\text {tr }}$, this inelastic displacement is smaller than the applied displacement since part of the overall deformation is elastic. The inset in Figure 3 shows the region of the yield surface where the phase transformation is most active, lying in the cone $\alpha \in\left[15^{\circ}, 60^{\circ}\right]$. Overall, the in-plane yield surface is highly anisotropic.

We now consider the resolved shear stress $\tau$ along the transformation direction $\mathbf{s}^{(1)}\left(\alpha_{\mathrm{tr}}=\right.$ $\left.19.5^{\circ}\right)$ computed as $^{1}$

$$
\tau=\Sigma:\left(\mathbf{s}^{(1)} \otimes \mathbf{n}^{(1)}\right)
$$

for applied loads at all angles $\alpha$ where transformation occurs as indicated in Figure 3 (note that $\left.\mathbf{m} \neq \mathbf{s}^{(1)}\right)$. As shown in Figure 4, all stress-displacement curves overlap except for $\alpha=180^{\circ}$ (at this precise angle, the simulation cell is overconstrained to accommodate shear deformation along one in-plane direction, see Appendix B, leading to higher stresses). The collapse of all stress-strain curves along the phase transformation direction indicates that the phase transformation activates as in Schmid's law. Therefore, in analogy to crystal plasticity, initiation of the phase transformation can be described by the Kuhn-Tucker conditions

$$
f=|\tau|-\tau_{c} \leq 0 \quad \dot{\varepsilon}_{\mathrm{tr}} \geq 0 \quad f \dot{\varepsilon}_{\mathrm{tr}}=0
$$

where $f$ is the transformation criterion, $\tau_{c}$ the critical resolved shear stress at which transformation starts (with different values for forward and reverse transformation) and $\dot{\varepsilon}_{\text {tr }}$ is the transformation rate (superimposed dot indicates the time derivative), which is proportional to the rate of change of the volume fraction of transforming material, as described in more detail in Appendix C. The third condition in Eq. (3) means that the interface moves only if the transformation criterion is satisfied, and vice-versa. Note that Eqs. (2) and (3) are the atomistic justification of the widely-used Patel and Cohen criterion for stress-assisted martensitic transformations [22].

\footnotetext{
${ }^{1}$ Here, all quantities related to the "shape deformation" tensor $\mathbf{P}^{(1)}$ from the crystallographic theory of martensite follow the notation in [12].
} 
This is a post-print of the following article: Maresca, F.; Kouznetsova, V.G.; Geers, M.G.D.; Curtin, W.A. Acta Materialia 2018,, 463-478.. The formal publication is available at http://dx.doi.org/10.1016/j.actamat.2018.06.028 @ 2018. This manuscript version is made available under the CC-BY-NC-ND 4.0 license http://creativecommons.org/licenses/by-nc-nd/4.0/

Figure 4 also confirms that the asymmetry in the fcc-bcc transformation behaviour is substantial. While the forward transformation is essentially spontaneous, the critical stress for driving the reverse bcc-to-fcc transformation $(\sim 340 \mathrm{MPa})$ is much larger than the stress needed to overcome the thermodynamic driving force $(\Delta E \simeq 11.25 \mathrm{meV})$ for the fcc-to-bcc transformation for the MEAM-T potential at $\mathrm{T}=0 \mathrm{~K}$. The thermodynamic energy difference can be overcome by the work done by an applied stress of $\tau_{\Delta E}=\Delta E / A b \simeq 130 \mathrm{MPa}$, where $b=2.52 \AA$ is the magnitude of the fcc Burgers vector and $A=\frac{\sqrt{3}}{2} b^{2}$ is the surface area per atom swept by the interface during transformation. The difference between $340 \mathrm{MPa}$ and $130 \mathrm{MPa}$ indicates that there is an additional energetic barrier inhibiting the reverse transformation.

The asymmetry in critical stress can be understood by considering the Peierls stresses of the interface dislocations. The interface structure in Figure 1b shows that when forward transformation occurs, the interface dislocations glide in the existing fcc lattice and transform it into bcc. Conversely, when the reverse transformation occurs, the interface dislocations glide in the bcc lattice. There are two sets of dislocations at the interface, namely bcc screws with kinks and fcc screw dislocations. The bcc screw dislocations with edge kinks move nearly athermally: the Peierls stress of a bcc edge dislocation at $\mathrm{T}=0 \mathrm{~K}$ is $3.25 \mathrm{MPa}( \pm 0.25 \mathrm{MPa})$ for Fe for this MEAM-T potential. Thus, the interface defect that might move with a non-negligible barrier is the fcc screw dislocation. This dislocation shares the same glide plane as a bec dislocation and has a similar Burgers vector and direction (at an angle $\varphi \leq 5.26^{\circ}$ ). We thus hypothesize that (i) the forward transformation occurs with the interface screw dislocation gliding effectively in the fcc lattice at a critical stress controlled by the Peierls stress for an fcc screw dislocation and, conversely, (ii) the reverse transformation occurs when this interface screw dislocation glides in the bcc lattice at a critical stress controlled by the Peierls stress for a bcc screw dislocation. This hypothesis quantitatively rationalizes the simulation results. For the MEAM-T Fe potential at $\mathrm{T}=0 \mathrm{~K}$, an fcc screw dislocation glides at $\tau_{P, \mathrm{fcc}}=90.5 \mathrm{MPa}( \pm 0.5 \mathrm{MPa})$ while the bcc screw dislocation glides at $\tau_{P, \mathrm{bcc}}=377.5 \mathrm{MPa}( \pm 0.5 \mathrm{MPa})$. According to our hypothesis, the critical stress for forward transformation, resolved on the habit plane at an angle $\theta$ with respect to the slip plane $(111)_{\mathrm{fcc}}$ along the direction of screw dislocation motion, should then be

$$
\tau_{\mathrm{fcc} \rightarrow \mathrm{bcc}}=\left(\tau_{P, f c c}-\tau_{\Delta E}\right) \cos \theta=-38.4 \mathrm{MPa}
$$

indicating that the transformation is spontaneous, i.e. requiring no applied stress. Indeed, the 
This is a post-print of the following article: Maresca, F.; Kouznetsova, V.G.; Geers, M.G.D.; Curtin, W.A. Acta Materialia 2018,, 463-478.. The formal publication is available at http://dx.doi.org/10.1016/j.actamat.2018.06.028 @ 2018. This manuscript version is made available under the CC-BY-NC-ND 4.0 license http://creativecommons.org/licenses/by-nc-nd/4.0/

interface is stabilized in simulations by introducing undetectable defects to study an interface which does not move after relaxation (see [12]). Furthermore, the reverse transformation should require a critical stress, resolved on the habit plane, of

$$
\tau_{\mathrm{bcc} \rightarrow \mathrm{fcc}}=\left(\tau_{P, b c c}+\tau_{\Delta E}\right) \cos \theta=497 \mathrm{MPa}
$$

Both stresses above are resolved along the screw dislocation direction, not the transformation direction $\alpha_{\mathrm{tr}}$. These stresses should thus be compared to the simulation stresses for $\tau_{\alpha=0^{\circ}}=-47 \mathrm{MPa}$ for forward transformation and $\tau_{\alpha=180^{\circ}}=490 \mathrm{MPa}$ for reverse transformation, respectively, which agrees well with the above estimate. As noted earlier, the simulations at $180^{\circ}$ are "anomalous" since shear deformation is constrained to occur only along the fcc screw dislocation direction. This specific boundary condition favours glide of the fcc screw dislocation, and inhibits the motion of the set of bcc screw dislocations (which glide transverse to the direction of the applied displacement). For loading conditions away from $\alpha=180^{\circ}$ the reverse transformation occurs at $\sim 80 \%$ lower $\tau_{\mathrm{bcc} \rightarrow \mathrm{fcc}}$, as can be readily verified by resolving $\tau_{\alpha=180^{\circ}}$ along the transformation direction $\alpha_{\mathrm{tr}}=19.5^{\circ}$, which yields $490 \mathrm{MPa} \cos \left(\alpha_{\mathrm{tr}}\right)=462 \mathrm{MPa}>370 \mathrm{MPa}$. The difference can be attributed to the interaction between the bcc screws with kinks and the fcc interface screws aligned with the steps, which appears to facilitate interface glide. The interaction between the multiple interface screws on different $(111)_{\text {fcc }}$ planes seems to play a minor role, since it is not visible in the $\alpha=180^{\circ}$ case.

This atomistic study of the mechanical response of the fcc-bcc interface reveals one origin of the well known fcc/bcc transformation asymmetry. That is, it is possible to obtain martensite upon quenching but not always possible to reverse the transformation [2]. This result is independent of the interatomic potential used here, since we have demonstrated that it is connected to glide of screw dislocations in fcc (forward transformation) or bcc (reverse transformation). While energy barriers for fcc screw glide are not high, it is well established that the energy barrier associated with bcc dislocation glide can be very large. While the forward transformation in pure defect-free Fe has a near-zero energy barrier that is overcome easily by the thermodynamic driving force alone (i.e. it is intrinsically athermal [12]), extrinsic phenomena such as alloying or pre-existing dislocations can provide some finite barrier to interface motion so that the fcc phase does not transform at zero stress in actual materials. For the reverse transformation, it is well-established that the bcc Peierls stress in pure Fe is quite high: DFT 
This is a post-print of the following article: Maresca, F.; Kouznetsova, V.G.; Geers, M.G.D.; Curtin, W.A. Acta Materialia 2018,, 463-478.. The formal publication is available at http://dx.doi.org/10.1016/j.actamat.2018.06.028 @ 2018. This manuscript version is made available under the CC-BY-NC-ND 4.0 license http://creativecommons.org/licenses/by-nc-nd/4.0/

calculations $[23,24]$ at $\mathrm{T}=0 \mathrm{~K}$ give 1.5-2.0 $\mathrm{GPa}$ and experiments at room temperature report $370 \mathrm{MPa}$ [25]. The present model, DFT, and experiments therefore agree on the existence of a high intrinsic barrier to bcc screw motion, and thus an intrinsically stress- and time-dependent reverse transformation. As for the forward transformation, the interaction of the interface dislocations with solutes and lattice dislocations and other defects would provide further increases to the barrier for interface motion.

\section{Transformation-induced plasticity (TRIP) model for Fe-C lath martensite mi- crostructures}

We now incorporate the atomistic findings into a continuum-scale TRIP model, which will then be applied to real martensitic microstructures [7]. While TRIP is usually associated with the plastic (slip) deformation in the surrounding microstructure induced by the transformation of metastable austenite, here the transformation itself provides an additional apparent plastic deformation. The passage from MD to continuum modeling is based on the knowledge, coming from the crystallographic theory of martensite [12], of the strain due to the phase transformation - the triplet $\left(m^{(1)}, \mathbf{s}^{(1)}, \mathbf{n}^{(1)}\right)$ - and the insight coming from Section 2, that the interface activates according to a Schmid law. Thus, the model does not need direct coupling to MD simulations, which enables the efficient simulation of microstructures at the micrometer scale - largely beyond MD capabilities.

In spite of the very small volume fractions of interlath austenite films retained between martensitic laths after quenching $[6,26]$, the results here will show that phase transformation in such thin nanoscale austenite can contribute significantly to the apparent macroscopic plasticity of real microstructures. This complements previous work wherein the contributions of the actual dislocation plasticity in the nanoscale austenite were evaluated [10]. Given the very limited thickness of the austenite films (few nm), dislocation-mediated plasticity in the austenite will not be modelled; instead we assume that any inelastic deformation in the austenite originates from the austenite to martensite phase transformation only.

\subsection{Lamellar model for nanolaminate martensite with films of austenite}

The TRIP model for martensite with thin films of austenite is based on the general framework proposed in [10], where lath martensite grains are modeled according to a two-scale 
This is a post-print of the following article: Maresca, F.; Kouznetsova, V.G.; Geers, M.G.D.; Curtin, W.A. Acta Materialia 2018,, 463-478.. The formal publication is available at http://dx.doi.org/10.1016/j.actamat.2018.06.028 @ 2018. This manuscript version is made available under the CC-BY-NC-ND 4.0 license http://creativecommons.org/licenses/by-nc-nd/4.0/

continuum approach. The framework is based on the observation [27] that lath martensite is characterized by a hierarchical microstructure consisting of multiple subgrains. Here, the word "subgrain" refers to all units related by crystallographic orientation relationship that form in lath martensite: crystallographic packets, blocks, and sub-blocks. All these units are related to one prior austenite grain. A complete description of the hierarchical microstructure of lath martensite is provided in [10]. When quenching to room temperature, within one single crystal fcc austenite grain, a polycrystalline microstructure forms which consists of up to 24 bcc crystallographic variants. The bcc variant spatial configurations are related to the parent fcc phase by the parallelism relation $\{111\}_{\text {fcc }} \|\{011\}_{\text {bcc }}$ between crystallographic planes and a small misorientation $\varphi \in\left[0^{\circ}, 5.26^{\circ}\right]$ between the close-packed directions $\langle\overline{1} 01\rangle_{\mathrm{fcc}}$ and $\langle\overline{1} \overline{1} 1\rangle_{\mathrm{bcc}}$. Each single variant is not a single crystal, but rather a collection of multiple single crystals. It is essential to recall that the small martensite crystals (laths) have an elongated, flat shape with a characteristic thickness approximately $100 \mathrm{~nm}$ forming stacks called "sub-blocks", where all martensite crystals share the same crystallographic orientation (the bcc variant) with respect to the austenite. The austenite decorates, in general, all boundaries of the stack in the form of thin films (down to $\sim 5 \mathrm{~nm}$ thick). Therefore, a "sub-block" of lath martensite is a bcc-fcc nanolaminate in which the interface normal is the habit plane $\mathbf{n}^{(1)}$, which can be predicted with the crystallographic theory of lath martensite [12]. In lath martensite microstructures, "blocks" are often observed, which are the regions where laths belonging to two different crystallographic variants form a single stack. The habit planes of these two variants are slightly different, and both experiments and crystallographic theory [12, 27] show that the misorientation between the average block interface and the single variant interface is $<7^{\circ}$. We therefore also model the "blocks" as nanolaminates, with a single interface normal that is equal to the average habit plane of the two constituent variants. Thus, a lath martensite microstructure is modeled as a collection of "sub-blocks" and "blocks", namely grains that share the same crystallography and interface orientation.

Following the above description of the microstructure of lath martensite as a collection of austenite film-martensite lath nanolaminates, we model the martensite laminate microstructure with an austenite volume fraction $(1-\xi)$ by expressing the laminate-level (L) deformation 
This is a post-print of the following article: Maresca, F.; Kouznetsova, V.G.; Geers, M.G.D.; Curtin, W.A. Acta Materialia 2018,, 463-478.. The formal publication is available at http://dx.doi.org/10.1016/j.actamat.2018.06.028 @ 2018. This manuscript version is made available under the CC-BY-NC-ND 4.0 license http://creativecommons.org/licenses/by-nc-nd/4.0/

gradient tensor $\mathbf{F}_{L}$ as

$$
\mathbf{F}_{L}=\xi \mathbf{F}_{M}+(1-\xi) \mathbf{F}_{A}
$$

which is the phase average of the total deformation gradient tensors in martensite laths $\mathbf{F}_{M}$ and austenite films $\mathbf{F}_{A}$, respectively. The first Piola-Kirchhoff stress $\mathbf{P}$ of the laminate is analogously expressed as

$$
\mathbf{P}_{L}=\xi \mathbf{P}_{M}+(1-\xi) \mathbf{P}_{A}
$$

The laminate geometry imposes constraints of interface compatibility and traction continuity at the block or sub-block interface, expressed as

$$
\begin{aligned}
\mathbf{F}_{M} \cdot\left(\mathbf{I}-\mathbf{n}^{(1)} \otimes \mathbf{n}^{(1)}\right) & =\mathbf{F}_{A} \cdot\left(\mathbf{I}-\mathbf{n}^{(1)} \otimes \mathbf{n}^{(1)}\right) \\
\mathbf{P}_{M} \cdot \mathbf{n}^{(1)} & =\mathbf{P}_{A} \cdot \mathbf{n}^{(1)}
\end{aligned}
$$

This system of equations is completed by specifying the single phase constitutive equations, i.e. $\mathbf{P}_{A}=\mathcal{F}\left(\mathbf{F}_{A}\right)$ and $\mathbf{P}_{M}=\mathcal{G}\left(\mathbf{F}_{M}\right)$.

Note that the compatibility condition expressed in Eq. $\left(8_{1}\right)$ is at the basis of the understanding of martensitic transformations: the habit plane $\mathbf{n}^{(1)}$ is the compatible, i.e. the common plane that is left unrotated and undistorted after transformation [28]. As for the traction continuity equation $(8)_{2}$, since the continuum viewpoint adopted here envisions the interface at a much larger scale than the atomistic analysis in Section 2, the average interface tractions in the neighboring phases have to balance to satisfy equilibrium.

\subsection{Phase transformation model for the austenite films}

For the austenite phase, we develop a kinematic model in which inelastic deformation arises from the phase transformation along the transformation direction rather than from fcc dislocation plasticity. This is the key difference between the present model and earlier work [10]. Thus, including elastic contributions, the total deformation gradient $\mathbf{F}_{A}$ of a single thin austenite layer that undergoes transformation can be split, as in standard plasticity models, into elastic $\mathbf{F}_{A, e}$ and inelastic $\mathbf{F}_{\text {tr }}$ contributions as

$$
\mathbf{F}_{A}=\mathbf{F}_{A, e} \cdot \mathbf{F}_{\mathrm{tr}}
$$

In the austenite phase, we assume the standard elastic constitutive law

$$
\overline{\mathbf{S}}_{A}=\mathbb{C}_{A}: \mathbf{E}_{A, e}
$$


This is a post-print of the following article: Maresca, F.; Kouznetsova, V.G.; Geers, M.G.D.; Curtin, W.A. Acta Materialia 2018,, 463-478.. The formal publication is available at http://dx.doi.org/10.1016/j.actamat.2018.06.028 @ 2018. This manuscript version is made available under the CC-BY-NC-ND 4.0 license http://creativecommons.org/licenses/by-nc-nd/4.0/

where $\overline{\mathbf{S}}_{A}$ is the pull-back of the Kirchhoff stress tensor $\boldsymbol{\tau}_{A}$ to the intermediate configuration, which is the configuration after applying the inelastic deformation. Also $\mathbf{E}_{A, e}=$ $\frac{1}{2}\left(\mathbf{F}_{A, e}^{T} \cdot \mathbf{F}_{A, e}-\mathbf{I}\right)$ is the elastic Green-Lagrange strain and $\mathbb{C}_{A}$ is the fourth order elasticity tensor.

The "transformation" contribution $\mathbf{F}_{\text {tr }}$ to the total deformation is calculated by defining an evolution law for its velocity gradient $\mathbf{L}_{\mathrm{tr}}:=\dot{\mathbf{F}}_{\mathrm{tr}} \cdot \mathbf{F}_{\mathrm{tr}}^{-1}$

$$
\mathbf{L}_{\mathrm{tr}}=\sum_{\alpha=1}^{n_{t}} \dot{\varepsilon}_{\mathrm{tr}}^{\alpha} \mathbf{s}^{(1), \alpha} \otimes \mathbf{n}^{(1), \alpha}
$$

where $n_{t}$ is the number of transformation systems within the austenite film; $\dot{\varepsilon}_{\mathrm{tr}}^{\alpha}$ is the transformation rate on the $\alpha$ th transformation system, $\mathbf{s}^{(1), \alpha}$ is the transformation direction, and $\mathbf{n}^{(1), \alpha}$ the habit (transformation) plane normal, defined on the initial body. Here, we consider only forward transformation systems, but the formulation is general and reverse transformation systems can be also included. For the lath martensite nanolaminate microstructure, there is one transformation system for a sub-block $(\alpha=1)$, and two for a block $(\alpha=1,2)$. In the sub-block, the transformation plane $\mathbf{n}^{(1)}$ normal coincides with the interface normal in Eq. (8), while in the block the interface normal is the average of $\mathbf{n}^{(1), 1}$ and $\mathbf{n}^{(1), 2}$.

The transformation rate is

$$
\dot{\varepsilon}_{\mathrm{tr}}^{\alpha}=\dot{\xi}_{\mathrm{tr}}^{\alpha} m^{(1)}
$$

where $\xi_{\mathrm{tr}}^{\alpha}$ is the transformed austenite fraction of system $\alpha$ with respect to the original austenite volume. In this work, to simplify the computational framework, it is assumed that the "transformed" austenite fraction $\xi_{\text {tr }}^{\alpha}$ does not correspond to an actual new phase but is rather used as a state variable to track the amount of phase transformation. This introduces a generally negligible error that scales with the austenite volume fraction $1-\xi_{0}$ (less than $5 \%$ in the examples representative microstructures treated below) and with the difference in elastic constants between austenite and martensite. In the above, $m^{(1)}, \mathbf{s}^{(1)}$ and $\mathbf{n}^{(1)}$ are the shape deformation (the "in-situ" transformation strain), the transformation direction and the habit plane normal, respectively, which are computed using the crystallographic theory of martensite [12].

Therefore, unlike previous TRIP models (e.g. [16]), the kinematic description of Equation (9) treats the transformation system $\mathbf{s}^{(1), \alpha} \otimes \mathbf{n}^{(1), \alpha}$ as a "slip system" in the austenite phase. Since the austenite films have a finite thickness, the apparent plasticity of the austenite evolves 
This is a post-print of the following article: Maresca, F.; Kouznetsova, V.G.; Geers, M.G.D.; Curtin, W.A. Acta Materialia 2018,, 463-478.. The formal publication is available at http://dx.doi.org/10.1016/j.actamat.2018.06.028 @ 2018. This manuscript version is made available under the CC-BY-NC-ND 4.0 license http://creativecommons.org/licenses/by-nc-nd/4.0/

only until the entire film is transformed. Therefore, for sub-blocks $\xi_{\operatorname{tr}} \leq \xi_{\max }=1$ while for blocks (assuming that the volume fraction of the two variants is equal) $\xi_{\text {tr }}^{\alpha} \leq \xi_{\max }=\frac{1}{2}$.

For the evolution of the transformation rate $\dot{\varepsilon}_{\mathrm{tr}}^{\alpha}$, we adopt the evolution law

$$
\dot{\varepsilon}_{\mathrm{tr}}^{\alpha}=\dot{\varepsilon}_{0}\left(\frac{\left|\tau^{\alpha}\right|}{\tau_{\mathrm{tr}}^{\alpha}}\right)^{\frac{1}{m_{A}}}
$$

where $\dot{\varepsilon}_{0}$ is a reference transformation rate, $m_{A}$ a transformation rate sensitivity parameter, $\tau_{\mathrm{tr}}^{\alpha}$ the critical transformation stress on the $\alpha$ th transformation system, and the resolved stress

$$
\tau^{\alpha}=\left\langle\left(\mathbf{F}_{A, e}^{T} \cdot \mathbf{F}_{A, e} \cdot \overline{\mathbf{S}}_{A}\right):\left(\mathbf{s}^{(1), \alpha} \otimes \mathbf{n}^{(1), \alpha}\right)\right\rangle
$$

where $\langle\cdot\rangle$ denote the Macauley brackets so that the forward transformation is activated only by a stress resolved along the positive transformation direction. The reference transformation rate $\dot{\varepsilon}_{0}$ and the transformation rate sensitivity parameter $m_{A}$ have no physical meaning here, but are adopted for computational convenience. Inverting the flow rule (13) results in $\tau^{\alpha}=$ $\tau_{\mathrm{tr}}^{\alpha}\left(\frac{\dot{\mathrm{t}}_{\mathrm{tr} \alpha}}{\dot{\varepsilon}_{0}}\right)^{m_{A}} \simeq \tau_{\mathrm{tr}}^{\alpha}$ as $m_{A} \rightarrow 0$ and $\dot{\varepsilon}_{\mathrm{tr}}^{\alpha}>0$, which shows that Eq. (13) is a regularization of the rate-independent phase transformation criterion expressed by Eq. (3).

Finally, for computational stabilization, we adopt a phenomenological evolution law for the critical stress for the transformation as

$$
\dot{\tau}_{\mathrm{tr}}^{\alpha}=h_{A, 0}\left(1-\frac{\tau_{\mathrm{tr}}^{\alpha}}{\tau_{A, \infty}}\right)^{a_{A}}\left|\dot{\varepsilon}_{\mathrm{tr}}^{\alpha}\right|
$$

where $h_{A, 0}$ is a "hardening" modulus, $\tau_{A, \infty}$ is a saturation value of the critical stress, and $a_{A}$ is a "hardening"-like parameter. These parameters have no physical meaning since there is no mechanism for hardening; parameters (see below) are thus chosen for numerical purposes. The key physical parameter is $\tau_{c}^{\alpha}$, the initial critical stress for forward transformation.

In summary, the fcc phase is treated as elastic with no dislocation plasticity, but with an apparent plasticity arising from the transformation. The transformed austenite is not treated as a new phase ("fresh" martensite) and so retains the austenite elastic properties. This simplification is expected to yield minor errors, scaling with the (small) elastic mismatch between austenite and martensite and the (very small) total volume fraction of the transforming austenite. Neglecting plasticity in the "fresh" martensite is reasonable since the thin austenite films are likely to contain more carbon than the surrounding martensite laths, making the "fresh" martensite stronger than the original martensite. 
This is a post-print of the following article: Maresca, F.; Kouznetsova, V.G.; Geers, M.G.D.; Curtin, W.A. Acta Materialia 2018,, 463-478.. The formal publication is available at http://dx.doi.org/10.1016/j.actamat.2018.06.028 @ 2018. This manuscript version is made available under the CC-BY-NC-ND 4.0 license http://creativecommons.org/licenses/by-nc-nd/4.0/

\subsection{Crystal plasticity model for the martensite laths}

The martensite lath phase is modeled as a bcc crystal with the same split of deformation gradient as

$$
\mathbf{F}_{M}=\mathbf{F}_{M, e} \cdot \mathbf{F}_{M, p}
$$

where $\mathbf{F}_{M, e}$ is the elastic deformation of the lath and $\mathbf{F}_{M, p}$ the plastic deformation. The elastic deformation follows Eq. (10) above. The plastic deformation is described using standard crystal plasticity based on the $\{110\}_{\text {bcc }}$ slip family. Note that both $\{110\}_{\text {bcc }}$ and $\{112\}_{\text {bcc }}$ slip are observed in Fe alloys [30] but crystallographic analysis in martensite microstructures [7] suggests that $\{110\}_{\mathrm{bcc}}$ slip is more favourable than $\{112\}_{\mathrm{bcc}}$. Previous work [10, 29] also demonstrated that the exact slip activity of martensite laths is less important than the inelastic deformation of the austenite films. We also do not include non-Schmid effects, based on conclusions in recent work $[30,31]$.

The evolution of the plastic deformation $\mathbf{F}_{M, p}$ is governed by the plastic velocity gradient $\mathbf{L}_{p}$

$$
\mathbf{L}_{p}=\sum_{\alpha=1}^{n_{s}} \dot{\gamma}^{\alpha} \mathbf{P}_{0}^{\alpha}
$$

where $\mathbf{P}_{0}^{\alpha}:=\mathbf{s}_{0}^{\alpha} \otimes \mathbf{n}_{0}^{\alpha}$ is the Schmid tensor of the $\alpha$ th slip system, $\mathbf{s}_{0}^{\alpha}$ is the slip direction and $\mathbf{n}_{0}^{\alpha}$ the slip normal, both defined in the initial body; $\dot{\gamma}^{\alpha}$ is the plastic slip rate on slip system $\alpha$; and $n_{s}=12$ is the number of slip systems considered here. The plastic slip rate $\dot{\gamma}^{\alpha}$ is determined via the visco-plastic slip law [53]

$$
\dot{\gamma}^{\alpha}=\dot{\gamma}_{0}\left(\frac{\left|\tau^{\alpha}\right|}{\tau_{y}^{\alpha}}\right)^{\frac{1}{m_{M}}} \operatorname{sign}\left(\tau^{\alpha}\right)
$$

where $\dot{\gamma}_{0}$ is a reference slip rate, $m_{M}$ is a strain rate sensitivity parameter, $\tau^{\alpha}$ the resolved shear stress on the $\alpha$ th slip system and $\tau_{y}^{\alpha}$ the current slip resistance. The resolved shear stress $\tau^{\alpha}$ is calculated according to the generalized Schmid law as

$$
\tau^{\alpha}=\left(\mathbf{F}_{M, e}^{T} \cdot \mathbf{F}_{M, e} \cdot \overline{\mathbf{S}}_{M}\right): \mathbf{P}_{0}^{\alpha}
$$

The current slip resistance $\tau_{y}^{\alpha}$ follows the hardening law

$$
\dot{\tau}_{y}^{\alpha}=\sum_{\beta=1}^{n_{s}} h^{\alpha \beta}\left|\dot{\gamma}^{\beta}\right|
$$


This is a post-print of the following article: Maresca, F.; Kouznetsova, V.G.; Geers, M.G.D.; Curtin, W.A. Acta Materialia 2018,, 463-478.. The formal publication is available at http://dx.doi.org/10.1016/j.actamat.2018.06.028 @ 2018. This manuscript version is made available under the CC-BY-NC-ND 4.0 license http://creativecommons.org/licenses/by-nc-nd/4.0/

where

$$
h^{\alpha \beta}=h_{M, 0}\left(1-\frac{\tau_{y}^{\alpha}}{\tau_{M, \infty}}\right)^{a_{M}} q^{\alpha \beta}
$$

with $q^{\alpha \beta}$ an interaction matrix for self and latent hardening, $h_{M, 0}$ an hardening modulus, $\tau_{M, \infty}$ the saturation value of the slip resistance, and $a_{M}$ an exponent setting the degree of nonlinearity of the hardening law. We take $q^{\alpha \alpha}=1$ and $q^{\alpha \beta}=q_{n}, \alpha \neq \beta . \tau_{0}^{\alpha}$ is the initial value of the slip resistance $\tau_{y}^{\alpha}$ for $\gamma^{\alpha}=0$.

\subsection{Lath martensite model configurations}

As Figure 5 shows, and as explained in more detail in [10], lath martensite is composed of different crystallographic variants (indicated by the letter V). Each variant is a parallel stack of stronger bcc $\alpha^{\prime}$ martensitic crystals (the laths) with an approximate average thickness of $100 \mathrm{~nm}$ and thin films of fcc retained austenite of average thickness $5 \mathrm{~nm}$. The crystallographic variants measured in [7] are labeled according to the Kurdjumov-Sachs (KS) orientation relationship, and are usually indexed pairwise and thus indicate blocks (see Appendix D). Unless a single variant is indicated, in which case the KS orientation is adopted for simulations, it is assumed that in one block the two variants have the same volume fraction, consistent with experiments [27, 32]. Table D.2 in the Appendix D lists the transformation direction and the normal for each variant, using the average orientation relationship $\varphi=2.5^{\circ}$ (between KS and Nishiyama-Wasserman $(\mathrm{NW}))[33]$ and austenite supersaturated with $\mathrm{C}$ [26] at the highest fcc/bcc lattice parameter ratio for which the fcc/bcc interface exists [12]. For this case, the transformation strain is $m^{(1)}=86.84 \%$ where $\left(m^{(1)}, \mathbf{s}^{(1)}, \mathbf{n}^{(1)}\right)$ are computed using the crystallographic theory of [12] for this composition and orientation relationship. We account for average sub-block and block interface orientations. Modeling of other steels with different compositions (e.g. Fe-Ni-Mn) would use the same crystallographic theory for the relevant alloy composition and orientation relationship. Note, that the framework adopted here is general: only the initial austenite volume fraction $1-\xi_{0}$ and the triplet $\left(m^{(1)}, \mathbf{s}^{(1)}, \mathbf{n}^{(1)}\right)$ coming from the crystallographic theory serve as the input, along with the material model parameters identified in Sections 3.2 and 3.3. For another alloy and microstructure, these input parameters will need to be determined. The model configurations are shown in Figure 5 and have been created using the EBSD data reported in [7]. The boundary conditions for these models are those used in [10]. The microstructure labeled as MP1 (Figure 5a) is characterized by crystallographic variants with fcc-bcc interfaces 
This is a post-print of the following article: Maresca, F.; Kouznetsova, V.G.; Geers, M.G.D.; Curtin, W.A. Acta Materialia 2018,, 463-478.. The formal publication is available at http://dx.doi.org/10.1016/j.actamat.2018.06.028 @ 2018. This manuscript version is made available under the CC-BY-NC-ND 4.0 license http://creativecommons.org/licenses/by-nc-nd/4.0/

mostly perpendicular to the loading direction. The microstructure labeled as MP2 (Figure 5b) is characterized by fcc-bcc interfaces mostly at $45^{\circ}$ with respect to the loading direction. These two configurations are selected from among those analyzed in [7] because the mechanical response of MP1 is expected to be dominated by the bcc lath plasticity having high yield strength while the response of MP2 is expected to have important contributions from the apparent plasticity due to the transformation in the austenite films, and hence deform more easily than MP1. The two model configurations are, therefore, ideal for testing the role of nanolaminate austenite films on the deformation of lath martensite microstructures. Model dimensions are $H=20 \mu m, T=20 \mu m, L_{1}=32.14 \mu m$ and $L_{2}=26.32 \mu m$. Tensile loading simulations are performed along the $\mathrm{X}$ direction at strain rate $\dot{\varepsilon}_{X X}=0.01 \mathrm{~s}^{-1}$, with free boundaries in the $\mathrm{Y}$ and $\mathrm{Z}$ directions, using the finite element method for these two microstructures. The faces perpendicular to $\mathrm{Y}$ and $\mathrm{Z}$ directions are left traction free because the configurations relate to the gauge section of uniaxial tensile specimens, while an average displacement is prescribed on the faces perpendicular to $\mathrm{X}$ direction.

\subsection{Microstructure simulation results}

As shown in [10], removing the fcc phase entirely and fitting the bcc parameters to the MP2 stress-strain curve leads to the wrong predicted trend for the MP1 stress-strain curve, as shown in the inset of Figure 6. The responses of the two microstructures are nearly identical, with just a small difference in flow stress, because the two bcc polycrystals have similar overall grain orientations. The inability to describe both MP1 and MP2 responses motivated the inclusion of fcc phase plasticity [10]. Here, the plasticity of the austenite is introduced as an "apparent" plasticity due to the inelastic deformation of the austenite films due to the phase transformation. The term "apparent" is used, since the phase transformation is not a standard dislocation-mediated phenomenon.

The constitutive parameters for the nanolaminate austenite/martensite system are chosen as follows. We use an fcc volume fraction $\left(1-\xi_{0}\right)=0.0476$ corresponding to $100 \mathrm{~nm}$ bcc laths and $5 \mathrm{~nm}$ interlath retained austenite. Since the films are very thin, we neglect changes in the overall martensite volume fraction due to transformation and treat the martensite volume fraction as a constant $\left(\xi=\xi_{0}\right)$. The remaining material model parameters are listed in Table 1 . We use the cubic elastic constants from [10] according to [34]. For the apparent fcc transformation 
This is a post-print of the following article: Maresca, F.; Kouznetsova, V.G.; Geers, M.G.D.; Curtin, W.A. Acta Materialia 2018,, 463-478.. The formal publication is available at http://dx.doi.org/10.1016/j.actamat.2018.06.028 @ 2018. This manuscript version is made available under the CC-BY-NC-ND 4.0 license http://creativecommons.org/licenses/by-nc-nd/4.0/

plasticity, small "hardening" is used just sufficient to provide computational stability while still reflecting an (almost) elastic/perfectly-plastic response as in Figure 4. Therefore, the only unknown fcc parameter is the critical stress for the forward transformation, which presumably depends on both the intrinsic dislocation Peierls stress for fcc and the interactions of the interface dislocation with $\mathrm{C}$ solutes and other possible defects in fcc. The value of the critical stress for transformation affects the onset of phase transformation, as detailed in Section 3.2. We assume that reverse transformation is inactive since it activates at much higher stress, and so no critical resolved shear stress is reported. For the selected fcc transformation stress, the bcc plasticity parameters (yield stress and hardening law) are fitted to reproduce the experimental stress-strain response for microstructure MP2. The overall set of plasticity parameters for fcc and bcc is not unique, but the values used here are reasonable and reflect the main effects of inclusion of fcc transformation plasticity.

With the chosen parameters fitted to microstructure MP2, Figure 6 shows the predicted stress-strain response for both microstructures along with the experimental data. The experimental trend of MP1 being stronger than MP2 is qualitatively reproduced. We have verified that, as long as the critical stress for the forward transformation $\tau_{c}$ is lower than the initial slip resistance in martensite $\tau_{0}$, specific adjustments of $\tau_{c} / \tau_{0}$ do not affect the qualitative result (i.e. MP1 is stronger than MP2).

Mismatch between simulations and experiments can be due to inaccurate model parameters, experimental accuracy (which appears limited, as seen in the elastic regime), and, especially, the assumption that crystallographic variants have exactly constant geometry along the specimen thickness. This restriction leads to constraining effects. In reality, FeC martensite shows a range of orientation relationships within the same material, with the average $\bar{\varphi}=2.5^{\circ}$ (adopted here) and a standard deviation $\Delta \varphi= \pm 1^{\circ}$ [33]. This implies a range of solutions for the habit planes $\left(\sim 8^{\circ}\right.$ to $12^{\circ}$, see Table 3 in [12]) and hence a range of solutions for the transformation systems. Incorporating a distribution of transformation systems among the different layers in the microstructure would facilitate transformation for some orientations. In addition, the present model constrains the "block" transformation to be the average over the transformation systems. Thus, real materials are subjected to less constraints with a spread of transformation directions as compared to the idealization here. Here, we fitted parameters to the MP2 response 
This is a post-print of the following article: Maresca, F.; Kouznetsova, V.G.; Geers, M.G.D.; Curtin, W.A. Acta Materialia 2018,, 463-478.. The formal publication is available at http://dx.doi.org/10.1016/j.actamat.2018.06.028 @ 2018. This manuscript version is made available under the CC-BY-NC-ND 4.0 license http://creativecommons.org/licenses/by-nc-nd/4.0/

Table 1: Material parameters for bcc martensite and fcc austenite single crystals.

\begin{tabular}{|c|c|c|}
\hline Initial slip resistance & $\tau_{0}$ & $370 \mathrm{MPa}$ \\
\hline Slip resistance saturation value & $\tau_{M, \infty}$ & $1500 \mathrm{MPa}$ \\
\hline Initial hardening rate & $h_{M, 0}$ & $500 \mathrm{MPa}$ \\
\hline Reference slip rate & $\dot{\gamma}_{0}$ & $0.01 s^{-1}$ \\
\hline Strain rate sensitivity & $m_{M}$ & 0.10 \\
\hline Hardening exponent & $a_{M}$ & 1.5 \\
\hline Ratio latent/self hardening & $q_{n}$ & 1.4 \\
\hline Elastic constant & $C_{M, 11}$ & $349 \mathrm{GPa}$ \\
\hline Elastic constant & $C_{M, 12}$ & $202.5 \mathrm{GPa}$ \\
\hline Elastic constant & $C_{M, 44}$ & $176.5 \mathrm{GPa}$ \\
\hline \multicolumn{3}{|l|}{ Material parameters, fcc } \\
\hline Initial forward transformation critical stress & $\tau_{c}$ & $200 \mathrm{MPa}$ \\
\hline Transformation resistance saturation value & $\tau_{A, \infty}$ & $260 \mathrm{MPa}$ \\
\hline Initial "hardening" rate & $h_{A, 0}$ & $250 \mathrm{MPa}$ \\
\hline Reference transformation rate & $\dot{\varepsilon}_{0}$ & $0.01 s^{-1}$ \\
\hline Transformation rate sensitivity & $m_{A}$ & 0.10 \\
\hline "Hardening" exponent & $a_{A}$ & 1.5 \\
\hline Elastic constant & $C_{A, 11}$ & $268.5 \mathrm{GPa}$ \\
\hline Elastic constant & $C_{A, 12}$ & $156.0 \mathrm{GPa}$ \\
\hline Elastic constant & $C_{A, 44}$ & $136.0 \mathrm{GPa}$ \\
\hline
\end{tabular}

within these constraints. Relaxing them would necessitate use of a stronger bcc phase to fit the MP2 stress-strain response. This stronger bcc response would then be reflected in an even stronger response for the MP1 microstructure, which is more dominated by the bcc behavior. Inclusion of these complex aspects can be addressed in future work, but would certainly contribute to decreasing the difference between simulated and experimental MP1 responses.

The strengthening of MP1 relative to MP2 is understood through examination of the slip 
This is a post-print of the following article: Maresca, F.; Kouznetsova, V.G.; Geers, M.G.D.; Curtin, W.A. Acta Materialia 2018,, 463-478.. The formal publication is available at http://dx.doi.org/10.1016/j.actamat.2018.06.028 @ 2018. This manuscript version is made available under the CC-BY-NC-ND 4.0 license http://creativecommons.org/licenses/by-nc-nd/4.0/

and transformation activity. Figure 7 shows the total slip activity in the bcc phase (laths) for both specimens at $1 \%$ global strain, computed as the sum of the absolute values of the slip on all active slip systems. Figure 8 shows the apparent plasticity due to transformation strain of the austenite films at the same $1 \%$ global strain both MP1 and MP2.

In microstructure MP1, some bcc plasticity occurs in all packets. In about one-third of the volume, the total slip reaches values close to $\sim 3 \%$ strain, which is the value predicted using the Taylor factor (ratio between total slip activity and applied strain) for bcc polycrystals featuring $\{110\}$ slip under tension [35]. The apparent plasticity of the austenite in MP1 is typically $5-10 \%$ transformation strain in about $1 / 4$ of the homogenized volume and very low values or $0 \%$ elsewhere. With the austenite volume fraction of only $\sim 5 \%$, the austenite deformation contributes to less than $0.5 \%$ strain to the overall nanolaminate. In microstructure MP2, the bcc lath plasticity is fairly uniformly distributed throughout the microstructure, but at levels less than 1\%, which is well below the typical Taylor factor prediction. In contrast, at only $1 \%$ global strain, the interlath austenite films accommodate up to $\sim 20 \%$ deformation in over $1 / 2$ of the homogenized volume. The apparent plasticity of the austenite is thus much more widespread in MP2 as compared to MP1. Overall, these results demonstrate that the local plastic response of the microstructures strongly depends on the relative orientation of the fcc-bcc interface with respect to the loading direction, with austenite films transforming, as expected, when oriented at $45^{\circ}$ with respect to the loading axis and not transforming when nearly perpendicular to the loading direction.

We now compare the predicted transformation activity with experiments [7]. In MP2, most of the apparent austenite plasticity is concentrated in blocks V21-24 and V19-22, consistent with the scanning white-light interferometry analysis in [7], where it is shown that already at $0.3 \%$ global strain "slip traces appeared in blocks V21 and V24. Protrusions also developed in the variants V19 and V22 and V20 and V23 in the vicinity of the packet and the block boundaries as the plastic strain increased". Although we do not predict much apparent austenite plasticity in V20-V23, this block is in-between the active blocks and thus our simulations are consistent with the observation of protrusions in the vicinity of the block boundaries. Simulations also confirm that the apparent austenite plasticity due to the variants transformed in the most active blocks starts to be exhausted above $4.0 \%$ global strain. At this strain level, about $50 \%$ of the 
This is a post-print of the following article: Maresca, F.; Kouznetsova, V.G.; Geers, M.G.D.; Curtin, W.A. Acta Materialia 2018,, 463-478.. The formal publication is available at http://dx.doi.org/10.1016/j.actamat.2018.06.028 @ 2018. This manuscript version is made available under the CC-BY-NC-ND 4.0 license http://creativecommons.org/licenses/by-nc-nd/4.0/

retained austenite of the active blocks has transformed. Overall, the model predictions agree qualitatively well with experiments, supporting our contention that a physical mechanism of austenite transformation observed at the atomic scale can contribute significantly to the overall deformation.

\section{Discussion}

Previous work [10] hypothesized that the thin austenite films retained at lath martensite boundaries contribute significantly to the aggregate lath martensite plastic deformation. Furthermore, it was assumed that the plastic deformation of nano-scale austenite films is due to the glide of the dislocations nearly parallel to the lath interfaces, since the interface plane is close to (although not exactly parallel to) a $\{111\}_{\text {fcc }}$ plane. Dislocation plasticity has more slip system orientations than the transformation systems, enabling a softer response and a higher ductility. On the other hand, at a film thickness of $l \sim 5 \mathrm{~nm}[6,26]$, and with interface orientations in FeC martensite at an angle $\theta \simeq 10^{\circ}$ from $(111)_{\text {fcc }}$ (habit plane $\approx(575)_{\text {fcc }}$ ), fcc dislocation loops are confined to a width $w \simeq \frac{l}{\sin \theta}=30 \mathrm{~nm}$. Similar to threading dislocations in multilayer nanoscale semiconductor devices [36], the critical stress required to extend such loops along the length of the thin austenite layer scales with the limiting loop radius $R=w / 2=15 \mathrm{~nm}$ as $\tau_{c} \sim \frac{\mu_{(111)} b}{w} \simeq 850 \mathrm{MPa}$, where $\mu_{(111)}=101 \mathrm{GPa}$ is the shear modulus of fcc austenite on the $\{111\}_{\text {fcc }}$ planes. More precise estimates for the stress to extend such fcc nanolayer loops depends on the actual line tension, and could be less than $850 \mathrm{MPa}$. However, the value would likely remain well above the values of $200 \mathrm{MPa}$ fitted here, the $\sim 250 \mathrm{MPa}$ fitted in [10] assuming fcc dislocation slip only, and the $\sim 300 \mathrm{MPa}$ estimated experimentally [7]. The present model thus complements the previous plasticity model in providing an additional austenite film inelastic deformation mechanism that does not depend on the film thickness and therefore is not affected by constraints on dislocation glide in the nanoscale structure. Likely, both mechanisms can contribute, with dislocation plasticity active in thicker layers and apparent plasticity due to transformation contributing more significantly in thin layers.

The present multi-scale TRIP model is not restricted to the specific FeC alloys studied in Section 3. It is applicable to a range of alloys including the most recent advanced high strength steels featuring a nanolaminate microstructure [1-4] with bcc martensite-fcc austenite as well as bcc bainite-fcc austenite (as demonstrated in [12]). This generality emerges because the 
This is a post-print of the following article: Maresca, F.; Kouznetsova, V.G.; Geers, M.G.D.; Curtin, W.A. Acta Materialia 2018,, 463-478.. The formal publication is available at http://dx.doi.org/10.1016/j.actamat.2018.06.028 @ 2018. This manuscript version is made available under the CC-BY-NC-ND 4.0 license http://creativecommons.org/licenses/by-nc-nd/4.0/

kinematic model of the transforming austenite is not material-specific, aside from involving an fcc-bcc transformation, and introduces only three material parameters: the two critical stresses $\tau_{c}$ for forward and reverse transformation and a maximum volume fraction $(1-\xi)$ of the transforming phase. These material parameters can be evaluated for any given system. For instance, $(1-\xi)$ is simply a geometry-dependent quantity measurable by phase mapping in SEM [3], or - when the austenite layers are few nanometers thin - by XRD experiments and TEM investigations [37, 38]. The critical stresses $\tau_{c}$ depend, in general, on alloy composition and dislocation densities in the two phases. But recall that we have correlated these quantities with the CRSS for screw dislocations in the fcc and bcc phases, respectively, which in turn are determined by solutes (interstitials - such as C - or in solid solution - e.g. Ni, Mn, Si, Cr) and by dislocations in the bulk that act as forest obstacles. Composition and temperature also contribute in determining the thermodynamic driving force for the fcc-to-bcc transformation, which also leads to the asymmetry in forward and reverse values of $\tau_{c}$. In Section $3, \tau_{c}$ for the forward transformation was based on experiments for a material with specific composition and properties [7]. Recently, experimental testing techniques have been developed that would be suitable for measuring $\tau_{c}$ for a specific alloy in a controlled microstructure with just a few laths [8]. Recent predictive solute-strengthening theories ([39, 40] for fcc; [41] for bcc) could be employed to determine $\tau_{c}$ for low dislocation densities, using first-principles studies of solute/dislocation interaction energies.

As pointed out in Section 2, this paper reveals one origin of the well known fcc/bcc transformation asymmetry. The work here also shows that the mechanical response of the fcc-bcc interface is strongly anisotropic, depending on the relative orientation of the stress state with respect to the transformation systems. This anisotropy underlies different (apparent) plasticity mechanisms. Therefore, both atomistic simulations and crystal plasticity simulations on configurations MP1 and MP2 show that whether austenite films transform, slip, or twin does not depend solely on the stacking fault energy (SFE), although the SFE is the determining factor in other systems such as high-manganese steels [42]. Also, the exact inelastic deformation mode (transformation, slip or twin) does not only depend on the grain size or film-thickness, as observed in the case of TRIP-maraging steels [3]. Our atomistic simulations have been performed for one film thickness, no variation of SFE was considered, and only the local loading direction 
This is a post-print of the following article: Maresca, F.; Kouznetsova, V.G.; Geers, M.G.D.; Curtin, W.A. Acta Materialia 2018,, 463-478.. The formal publication is available at http://dx.doi.org/10.1016/j.actamat.2018.06.028 @ 2018. This manuscript version is made available under the CC-BY-NC-ND 4.0 license http://creativecommons.org/licenses/by-nc-nd/4.0/

has been varied. Nonetheless, we find that the local stress state (at the level of crystallographic blocks) relative to the interface orientation has a huge effect on the apparent plastic phenomenon (Figure 3) and should be accounted for in experimental analyses. Furthermore, when austenite films are very thin (as it is the case for $\mathrm{FeC}$ ), it is often questioned whether they transform at all [38]. Hence, the fact that retained austenite can be found after some plastic strain does not imply that the austenite did not transform anywhere in the specimen: orientation dependence plays a key role in austenite transformation activity.

The present simulations on $\mathrm{FeC}$ lath martensite are also consistent with other experimental observations based on mechanical testing of lath martensite. Reference [43] reports that austenite films can no longer be found after 10\% cold rolling. Reference [44] reports exhaustion at $4 \%$ average tensile strain. Our simulations show that exhaustion of austenite starts around $4 \%$ strain (Section 4.4), consistent with these observations. Our findings are further consistent with recent experiments on low carbon, lath martensite [8] that reveal high plastic deformation localized at the lath interfaces in steels having a composition and processing that trigger thin retained austenite films at lath boundaries [6, 26]. A similar prevalence of plastic deformation parallel to the lath interface in martensitic steels was also reported in earlier works [45].

Finally, we compare our results on the transformation asymmetry with experiments. Experimentally, austenite reversion from martensite can be obtained by annealing [2, 46, 47], which makes austenite thermodynamically more favourable than martensite. This drives the reverse transformation. Our results indeed show that the reverse transformation is controlled by the high thermally-activated Peierls barrier for bcc screw glide, leading to the thermally-activated reversion, consistent with the observed time and temperature dependence of reversion. Under annealing at temperature $T$, the stress driving the transformation is $\tau_{T}=\Delta E(T) / A b$ and the transformation rate $r$ would then follow a typical Kocks-Mecking-type form of $r=$ $r_{o} e^{-\frac{\Delta E_{0}}{k T}}\left(\left(1-\left(\tau(T) / \tau_{y 0}\right)^{q}\right)^{p}\right.$ where $\Delta E_{0}$ and $\tau_{y 0}$ are the zero-temperature energy barrier and flow stress, respectively, and $p$ and $q$ are parameters associated with the precise flow mechanism of the dislocations. At typical annealing temperatures, it is expected that $\tau_{T}<<\tau_{y 0}$, making the reverse transformation quite slow. Moreover, experiments even show that the transformation is not always clearly "reversible". Reversibility is mostly observed for austenite films, rather than "blocky" austenite islands [46]. In [2], some austenite films grow upon tempering in accor- 
This is a post-print of the following article: Maresca, F.; Kouznetsova, V.G.; Geers, M.G.D.; Curtin, W.A. Acta Materialia 2018,, 463-478.. The formal publication is available at http://dx.doi.org/10.1016/j.actamat.2018.06.028 @ 2018. This manuscript version is made available under the CC-BY-NC-ND 4.0 license http://creativecommons.org/licenses/by-nc-nd/4.0/

dance with the orientation relationship, while others grow without exhibiting any orientation relationship with the neighboring martensite laths. Thus, the rate of the thermodynamicallydriven reverse transformation might be such that there is sufficient time for diffusive formation of austenite.

\section{Concluding remarks}

This paper has investigated the atomic scale mechanical response of the fcc-bcc interface having a structure consistent with experiments [12]. Simulations reveal a high anisotropy of the mechanical response of the fcc-bcc bicrystal with respect to the applied load, with an asymmetry for loading along the transformation direction that can be rationalized through the motion of the interface dislocations in the forward and reverse transformation directions as if they are in the fcc and bcc phase, respectively. Overall, the phase transformation by interface motion is activated according to a Schmid-type behaviour, confirming the broadly used Patel-Cohen criterion for martensite transformation [22]. The atomistic simulations have guided the formulation of a two-scale continuum TRIP model for austenite/martensite nanolaminate microstructures, where the inelastic deformation of the austenite films is described by the kinematics of the phase transformation via an apparent plasticity model. The nanolaminate model has then been used to simulate lath martensite microstructures with thin retained austenite films in Fe-C alloys. The model simulations including the effect of the transformation strain to the apparent plasticity of the microstructures confirm that the phase transforming films can contribute substantially to the observed plastic response of the overall martensitic microstructure, complementing the mechanism of austenitic dislocation plasticity envisioned previously in [10]. The present results are also consistent with multiple experimental observations. More broadly, the model provides a consistent multi-scale framework relating atomistic structure and mechanical response to large-scale microstructural mechanical behavior. The model may thus assist in the understanding and design of alloy steels having a nanolaminate austenite/martensite microstructure [1-5].

\section{Acknowledgements}

F.M. and W.A.C. acknowledge support of this work through a European Research Council Advanced Grant, Predictive Computational Metallurgy, ERC grant agreement no. 339081 - 
This is a post-print of the following article: Maresca, F.; Kouznetsova, V.G.; Geers, M.G.D.; Curtin, W.A. Acta Materialia 2018,, 463-478.. The formal publication is available at http://dx.doi.org/10.1016/j.actamat.2018.06.028 @ 2018. This manuscript version is made available under the CC-BY-NC-ND 4.0 license http://creativecommons.org/licenses/by-nc-nd/4.0/

PreCoMet.

\section{References}

[1] De Moor, E., Lacroix, S., Clarke, A., Penning, J., Speer, J.G. (2008) Effect of retained austenite stabilized via quench and partitioning on the strain hardening of martensitic steels. Metall. Mater. Trans. A 39:2586-2595.

[2] Raabe, D., Sandlöbes, S., Millán, J., Ponge, D., Assadi, H., Herbig, M., Choi, P.-P. (2013) Segregation engineering enables nanoscale martensite to austenite phase transformation at grain boundaries: A pathway to ductile martensite. Acta Mater. 61:6132-6152.

[3] Wang, M.-M., Tasan, C.C., Ponge, D., Kotska, A., Raabe, D. (2014) Smaller is less stable: Size effects on twinning vs. transformation of reverted austenite in TRIP-maraging steels. Acta Mater. 79:268-281.

[4] Sun, W.W., Wu, Y.X., Yang, S.C., Hutchinson, C.R. (2018) Advanced high strength steel (AHSS) development through chemical patterning of austenite. Scripta Mater. 146:60-63.

[5] Wang, M.-M., Tasan, C.C., Koyama, M., Ponge, D., Raabe, D. (2015) Enhancing hydrogen embrittlement resistance of lath martensite by introducing nano-films of interlath austenite. Metall. Mater. Trans. A 46:3797-3802.

[6] Sandvik, B.P.J., Wayman, C.M. (1983) Crystallography and substructure of lath martensite formed in cabon steels. Metallography 16:199-227.

[7] Mine, Y., Hirashita, K., Takashima, H., Matsuda, M., Takashima, K. (2013) Micro-tension behaviour of lath martensite structures of carbon steel. Mater. Sci. Eng. A 560:535-544.

[8] Du, C., Hoefnagels, J.P.M., Vaes, R., Geers, M.G.D. (2016) Plasticity of lath martensite by sliding of substructure boundaries. Scripta Mater. 120:37-40.

[9] Maresca, F., Kouznetsova, V.G., Geers, M.G.D. (2014) On the role of interlath retained austenite in the deformation of lath martensite. Model. Simul. Mater. Sci. Eng. 22:045011.

[10] Maresca, F., Kouznetsova, V.G., Geers, M.G.D. (2014) Subgrain lath martensite mechanics: A numerical-experimental analysis. J. Mech. Phys. Solids 73:69-83. 
This is a post-print of the following article: Maresca, F.; Kouznetsova, V.G.; Geers, M.G.D.; Curtin, W.A. Acta Materialia 2018,, 463-478.. The formal publication is available at http://dx.doi.org/10.1016/j.actamat.2018.06.028 @ 2018. This manuscript version is made available under the CC-BY-NC-ND 4.0 license http://creativecommons.org/licenses/by-nc-nd/4.0/

[11] Maresca, F., Kouznetsova, V.G., Geers, M.G.D. (2016) Predictive modeling of interfacial damage in substructured steels: application to martensitic microstructures. Modelling Simul. Mater. Sci Eng. 24:025006.

[12] Maresca, F., Curtin, W.A. (2017) The austenite/lath martensite interface in steels: Structure, athermal motion, and in-situ transformation strain revealed by simulation and theory. Acta Mater. 134:302-323.

[13] Wakasa, K., Wayman, C.M. (1981) The morphology and crystallography of Ferrous lath martensite. Studies of Fe-20\%Ni-5 \%Mn III. Surface relief, the shape strain and related features. Acta Metall. 29:1013-1028.

[14] Sandvik, B.P.J., Wayman, C.M. (1983) Characteristics of lath martensite: Part III. Some theoretical considerations. Metall. Trans. A 14:835-844.

[15] Bhadeshia, H.K.D.H. (2002) TRIP-assisted steels? ISIJ Int. 42:1059-1060.

[16] Kouznetsova, V.G., Geers, M.G.D. (2008) A multi-scale model of martensitic transformation plasticity. Mech. Mater. 40:641-657.

[17] Plimpton, S. (1995) Fast parallel algorithms for short-range molecular dynamics. J. Comp. Phys. 117:1-19. Available at: http://lammps.sandia.gov/

[18] Lee, T., Baskes, M.I., Valone, S.M., Doll, J.D. (2012) Atomistic modeling of thermodynamic equilibrium and polymorphism of iron. J. Phys.: Condens. Matter 24:225404.

[19] Stukowski, A. (2009) Visualization and analysis of atomistic simulation data with OVITO the Open Visualization Tool. Model. Simul. Mater. Sci. Eng. 18:015012.

[20] Stukowski, A. (2012) Structure identification methods for atomistic simulations of crystalline materials. Model. Simul. Mater. Sci. Eng. 20:045021.

[21] Warner, D.H., Molinari, J.F. (2008) Effect of normal loading on grain boundary migration and sliding in copper. Modelling Simul. Mater. Sci Eng. 16:075007.

[22] Patel, J.R., Cohen, M. (1953) Criterion for the action of applied stress in the martensitic transformation. Acta Metall. 1:531-538. 
This is a post-print of the following article: Maresca, F.; Kouznetsova, V.G.; Geers, M.G.D.; Curtin, W.A. Acta Materialia 2018,, 463-478.. The formal publication is available at http://dx.doi.org/10.1016/j.actamat.2018.06.028 @ 2018. This manuscript version is made available under the CC-BY-NC-ND 4.0 license http://creativecommons.org/licenses/by-nc-nd/4.0/

[23] Dezerald, L., Proville, L., Ventelon, L., Willaime, F., Rodney, D. (2015) First-principles prediction of kink-pair activation enthalpy on screw dislocations in bcc transition metals: V, Nb, Ta, Mo, W, and Fe. Phys. Rev. B 91:094105

[24] Dragoni, D., Daff, T.D., Csányi, G., Marzari, N. (2017) Achieving DFT accuracy with a machine-learning potential: themomechanics and defects in bcc ferromagnetic iron. ArXiv preprint:1706.10229v1

[25] Kuramoto, E., Aono, Y., Kitajima, K. (1979) Thermally activated slip deformation of high purity iron single crystals between 4.2 K and 300 K. Scripta Metall. 13:1039-1042.

[26] Morito, S., Oh-Ishi, K., Hono, K., Ohba, T. (2011) Carbon enrichment in retained austenite films in low carbon lath martensite steels. ISIJ Int. 51:1200-1202.

[27] Morito, S., Tanaka, H., Konishi, R., Furuhara, T., Maki, T. (2003) The morphology and crystallography of lath martensite in Fe-C alloys. Acta Mater. 51:1789-1799.

[28] Bhadeshia, H.K.D.H., Wayman, C.M. (2014) Phase transformations, nondiffusive, in: Physical Metallurgy, 5th edn. (D. Laughlin, K. Hono Eds.), Elsevier, 1021-1072.

[29] Maresca, F., Kouznetsova, V.G., Geers, M.G.D. (2016) Reduced crystal plasticity for materials with constrained slip activity. Mech. Mater. 92:198-210.

[30] Du, C., Maresca, F., Geers, M.G.D., Hoefnagels, J.P.M. (2018) Ferrite slip system activation investigation by uniaxial micro-tensile tests and simulations. Acta Mater. 146:314-327.

[31] Dezerald, L., Rodney, D., Clouet, E., Ventelon, L., Willaime, F. (2016) Plastic anisotropy and dislocation trajectory in BCC metals. Nature Comm. 7:11695.

[32] Morito, S., Huang, X., Furuhara, T., Maki, T., Hansen, N. (2006) The morphology and crystallography of lath martensite in alloy steels. Acta Mater. 54:5323-5331.

[33] Kelly, P.M., Jostsons, A., Blake, R.G. (1990) The orientation relationship between lath martensite and austenite in low carbon, low alloy steels. Acta Metall. Mater. 38:1075-1081.

[34] Turteltaub, S., Suiker, A. (2005) Transformation-induced plasticity in ferrous alloys. J. Mech. Phys. Solids 53:1747-1788. 
This is a post-print of the following article: Maresca, F.; Kouznetsova, V.G.; Geers, M.G.D.; Curtin, W.A. Acta Materialia 2018,, 463-478.. The formal publication is available at http://dx.doi.org/10.1016/j.actamat.2018.06.028 @ 2018. This manuscript version is made available under the CC-BY-NC-ND 4.0 license http://creativecommons.org/licenses/by-nc-nd/4.0/

[35] Rosenberg, J.M., Piehler, H.R. (1971) Calculation of the Taylor factor and lattice rotations for bcc metals deforming by pencil glide. Metall. Trans. 2:257-259.

[36] Freund, L.B. (1993) The mechanics of dislocations in strained-layer semiconductor materials. Adv. App. Mech. 30:1-66.

[37] Jimenez-Melero, E., van Dijk, N.H., Zhao, L., Sietsma, J., Offerman, S.E., Wright, J.P., van der Zwaag, S. (2007) Characterization of individual retained austenite grains and their stability in low-alloyed TRIP steels. Acta Mater. 55:6713-6723.

[38] Xiong, X.C., Chen, B., Huang, M.X., Wang, J.F., Wang, L. (2013) The effect of morphology on the stability of retained austenite in a quenched and partitioned steel. Scripta Mater. 68:321-324.

[39] Leyson, G.P.M., Curtin, W.A., Hector, L.G. Jr., Woodward, C. (2010) Quantitative prediction of solute strengthening in aluminium alloys. Nature Mater. 9:750-755.

[40] Varvenne, C., Luque, A., Curtin, W.A. (2016) Theory of strengthening in fcc high entropy alloys. Acta Mater. 118:164-176.

[41] Trinkle, D.R., Woodward, C. (2005) The chemistry of deformation: How solutes soften pure metals. Science 310:1665-1667.

[42] Allain, S., Chateau, J.-P., Bouaziz, O., Migot, S., Guelton, N. (2004) Correlations between the calculated stacking fault energy and the plasticity mechanisms in Fe-Mn-C alloys. Mater. Sci. Eng. A 387-389:158-162.

[43] Morito, S., Ohba, T., Das, A.K., Hayashi, T., Yoshida, M. (2013) Effect of solution carbon and retained austenite films on the development of deformation structures of low-carbon lath martensite. ISIJ Int. 12:2226-2232.

[44] Morsdorf, L., Jeannin, O., Barbier, D., Mitsuhara, M., Raabe, D., Tasan, C.C. (2016) Multiple mechanisms of lath martensite plasticity. Acta Mater. 121:202-214.

[45] Michiuchi, M., Nambu, S., Ishimoto, Y., Inoue, J., Koseki, T. (2009) Relationship between local deformation behavior and crystallographic features of as-quenched lath martensite during uniaxial tensile deformation. Acta Mater. 57:5283-5291. 
This is a post-print of the following article: Maresca, F.; Kouznetsova, V.G.; Geers, M.G.D.; Curtin, W.A. Acta Materialia 2018,, 463-478.. The formal publication is available at http://dx.doi.org/10.1016/j.actamat.2018.06.028 @ 2018. This manuscript version is made available under the CC-BY-NC-ND 4.0 license http://creativecommons.org/licenses/by-nc-nd/4.0/

[46] Zhang, X., Miyamoto, G., Toji, Y., Nambu, S., Koseki, T., Furuhara, T. (2018) Orientation of austenite reverted from martensite in Fe-2Mn-1.5Si-0.3C alloy. Acta Mater. 144:601-612.

[47] Ding, R., Dai, Z., Huang, M., Yang, Z., Zhang, C., Chen, H. (2018) Effect of pre-existed austenite on austenite reversion and mechanical behaviour of an Fe-0.2C-8Mn-2Al medium Mn steel. Acta Mater. In press.

[48] Abeyaratne, R., Knowles, J.K. On the driving traction acting on a surface of strain discontinuity in a continuum. J. Mech. Phys. Solids 38:345-360.

[49] Abeyaratne, R., Knowles, J.K. Kinetic relations and the propagation of phase boundaries in solids. Arch. Rational Mech. Anal. 114:119-154.

[50] Gurtin, M.E., Struthers, A. (1990) Multiphase thermomechanics with interfacial structure 3. Evolving phase boundaries in the presence of bulk deformation. Arch. Rational Mech. Anal. 112:97-160.

[51] Fischer, F.D., Berveiller, M., Tanaka, K. Oberaigner, E.R. (1994) Continuum mechanical aspects of phase transformations in solids. Arch. App. Mech. 64:54-85.

[52] Levitas, V.I. (1998) Thermomechanical theory of martensitic phase transformations in inelastic materials. Int. J. Solids Structures 35:889-940.

[53] Hutchinson, J.W. (1976) Bounds and self-consistent estimates for creep of polycrystalline materials. Proc. R. Soc. Lond. A 348:101-127.

[54] Guo, Z., Lee, C.S., Morris Jr., J.W. (2004) On coherent transformations in steel. Acta Mater. 52:5511-5518.

\section{Appendix A. Bicrystal construction and applied loading}

The bcc-fcc bicrystal of Section 2 has been constructed according to the procedure detailed in [12], namely by shifting the initial relative configuration of fcc and bcc with respect to an in-plane 10 x 10 grid, relaxing the system and selecting the minimum energy configuration (see [12]). Since there is a small energy difference $(\sim 10 \mathrm{meV} /$ atom $)$ that favours bcc over fcc, some initial configurations would consume the fcc phase by interface glide during the relaxation 
This is a post-print of the following article: Maresca, F.; Kouznetsova, V.G.; Geers, M.G.D.; Curtin, W.A. Acta Materialia 2018,, 463-478.. The formal publication is available at http://dx.doi.org/10.1016/j.actamat.2018.06.028 @ 2018. This manuscript version is made available under the CC-BY-NC-ND 4.0 license http://creativecommons.org/licenses/by-nc-nd/4.0/

process. Therefore, in order to study the interface structure and mobility, we select the interface with minimum energy, among those interfaces that do not move spontaneously. This procedure introduces undetectable defects that pin the interface at $\mathrm{T}=0 \mathrm{~K}$. The effect of these defects (slight atomic perturbations $<<b$ ) is minor, as the forward transformation can be activated by a slight stress perturbation (less than $1 \mathrm{MPa}$ ).

The system is loaded under multiple in-plane shear directions, by imposing a global affine shear deformation to the simulation cell. Strain-controlled loading is used. Shear deformation is applied parallel to the interface, while preserving a $3 \mathrm{GPa}$ hydrostatic pressure to simulate growth in constrained environment ${ }^{2}$. Simulations are performed at $\mathrm{T}=0 \mathrm{~K}$. Strain increments are applied by tilting (shearing) the simulation box along $\mathrm{YZ}$ and $\mathrm{XZ}$, therefore parallel to the interface plane Z:

$$
\begin{aligned}
& \text { tilt }_{\mathrm{YZ}}=\gamma_{0} \cos \alpha \\
& \text { tilt }_{\mathrm{XZ}}=\gamma_{0} \sin \alpha
\end{aligned}
$$

with $\alpha \in\left[-180^{\circ}, 180^{\circ}\right]$. The angle $\alpha$, defining the shear direction, is calculated with reference to $\mathrm{Y} \|[\overline{1} 01]_{\mathrm{fcc}}$ direction $\left(\alpha=0^{\circ}\right)$, and it is positive for clockwise rotations on the $\mathrm{Z} \|(111)_{\mathrm{fcc}}$ plane, towards $\mathrm{X} \|[\overline{1} 2 \overline{1}]_{\mathrm{fcc}}$. The strain increments are $\Delta \gamma=2.5 \cdot 10^{-4}$. The system energy is minimized at each strain level to achieve the associated equilibrium configuration, and the procedure (strain increment and minimization) is repeated along each loading direction $\alpha$ until significant deformation (corresponding to several Burgers vectors) is accommodated inelastically. The origin of the stress fluctuations in Figures 2 and 4 is the presence of the weak pinning points that make such interface metastable as discussed above. This Molecular Statics procedure ensures that the results are not influenced by time/rate dependent effects.

\section{Appendix B. Details on reverse transformation and cyclic loading}

This Appendix reports the atomistic details of the reverse transformation treated in Section 2, along with cyclic loading results. We first consider in detail the motion of each inter-

\footnotetext{
${ }^{2}$ The value of $3 \mathrm{GPa}$ accounts for the bulk moduli of the phases (close to $200 \mathrm{GPa}$ ) and a volume change due to transformation of approximately 1-2\%. It turns out that this hydrostatic pressure does not affect the interface structure [12], nor the interface activation (which is controlled essentially by shear, namely deviatoric stress).
} 
This is a post-print of the following article: Maresca, F.; Kouznetsova, V.G.; Geers, M.G.D.; Curtin, W.A. Acta Materialia 2018,, 463-478.. The formal publication is available at http://dx.doi.org/10.1016/j.actamat.2018.06.028 @ 2018. This manuscript version is made available under the CC-BY-NC-ND 4.0 license http://creativecommons.org/licenses/by-nc-nd/4.0/

face ledge for the case of bcc to fcc transformation. Like in the forward transformation, the $(111)_{\text {fcc }} \|(011)_{\text {bcc }}$ ledges preserve the local symmetry structure while gliding - the reverse transformation mechanism is glissile -. However, the mechanism is not athermal, as it requires a significant barrier to overcome. Considering the details of the interface, two ledges (out of 6 in the simulation cell) behave differently, as shown in Fig. B.9. On these ledges, the local interface structure changes: the interface ledge size shrinks about $20 \%$ in width (from $\sim 2 \mathrm{~nm}$ to $\sim 1.6 \mathrm{~nm}$, see Fig. B.9 label B), and the core of the bcc edge-character kinks, which can be compact when getting close to fcc, needs extra energy to spread along $[1 \overline{1} 1]_{\text {bcc }}$ direction and glide into bcc (see Fig. B.9 label A).

The core of the bcc edge-character kinks in the proximity of the interface is compact and tends to spread on $[100]_{\mathrm{bcc}}$, instead of the $[1 \overline{1} 1]_{\mathrm{bcc}}$ slip direction, because these two bcc directions are close to two fcc slip directions, $[0 \overline{1} 1]_{\mathrm{fcc}}$ and $[\overline{1} 10]_{\mathrm{fcc}}$ respectively. It is therefore the influence of the neighboring fcc lattice which favors spread of the bcc kink cores along a direction other than $[1 \overline{1} 1]_{\mathrm{bcc}}$. However, the same kink cores do not rearrange during forward transformation. This can be understood in terms of defect ordering with respect to transformation. When forward transformation is activated, the bcc kinks glide towards the interface to restore compatibility between the fcc and bcc misoriented crystals, and the screws propagate next to produce the correct $(011)_{\text {bcc }}$ stacking sequence. When reverse transformation takes place, the kinks cannot propagate further into bcc (there is no incompatibility to compensate for in front of them). Therefore, screws move first until kinks are sufficiently close to the fcc crystal, where their gliding would contribute to interface compatibility. This mechanism explains both the anomalous spread of $[1 \overline{1} 1]_{\mathrm{bcc}}$ and the observed shrinkage of the interface ledges. The shrinkage of the screw cores makes them closer to bcc screws, which is consistent with the observation that the stress controlling reverse transformation is comparable with the Peierls stress of a screw dislocation in bcc.

After reverse transformation is activated, if the system is further sheared in the opposite direction (favouring forward transformation) the bicrystal unloads elastically until $\sim 0 \mathrm{MPa}$ stress is reached. Then, forward transformation occurs. Figure B.10 reports in solid lines the cyclic stress-strain behaviour of the bicrystal.

The mechanical response is typical one for an elastic-perfectly plastic material, with elastic 
This is a post-print of the following article: Maresca, F.; Kouznetsova, V.G.; Geers, M.G.D.; Curtin, W.A. Acta Materialia 2018,, 463-478.. The formal publication is available at http://dx.doi.org/10.1016/j.actamat.2018.06.028 @ 2018. This manuscript version is made available under the CC-BY-NC-ND 4.0 license http://creativecommons.org/licenses/by-nc-nd/4.0/

loading/unloading followed by plastic deformation at constant stress. Also shown is the cyclic response (dashed line) for the case in which the load is applied along $\alpha=180^{\circ}$. In this case, reverse transformation kicks in at much higher stress (490 MPa), and subsequently hardens to an even higher stress $>600 \mathrm{MPa}$. As analyzed in Section 2, the first critical stress equals the stress required to move a screw dislocation in bcc. The reason for the subsequent hardening is that for this case (the only one among all directions probed where bcc to fcc transformation occurs) the interface kinks start crossing the interface from bcc to fcc, because the screw can move at its Peierls stress. One ledge where this phenomenon occurs is shown in Figure B.11.

Once the kink defects cross the screws towards fcc phase, they act as pinning sites for forward transformation, which explains the remarkable stress jump at the end of the elastic unloading (blue dashed line in Figure B.10). The occurrence of kink crossing and consequent interface self-pinning is likely due to the specific applied displacement boundary conditions (see also Appendix A) at $\alpha=180^{\circ}$, which constrain the bicrystal to deform along $[\overline{1} 01]_{\text {fcc }}$ without breaking any symmetry along $[\overline{1} 2 \overline{1}]_{\mathrm{fcc}}$. This explains why kink crossing never happens for all other load cases, where $\alpha \neq 180^{\circ}$.

\section{Appendix C. Details on the conditions for phase transformation through interface propagation in laminate microstructures}

This Appendix shows the connection between the Kuhn-Tucker conditions for interface propagation, based on atomistic simulation of an actual fcc-bcc interface, and the well established general continuum-mechanics theory for phase transformations through moving boundaries in laminate microstructures. This theory was first formulated in the early ' 90 s within the small deformation setting by Abeyaratne and Knowles [48, 49] and Gurtin and Struthers [50], who provided also a finite deformation formulation. Theories [48-50] provided the general framework for the development of all later continuum models of phase transformation in solids (e.g. $[16,51,52]$ to mention only a few).

By expressing the overall dissipation due to the phase transformation for the case of a planar interface in both small and large strains, we demonstrate the connection between Eq. (3) and the long-standing continuum theory. 
This is a post-print of the following article: Maresca, F.; Kouznetsova, V.G.; Geers, M.G.D.; Curtin, W.A. Acta Materialia 2018,, 463-478.. The formal publication is available at http://dx.doi.org/10.1016/j.actamat.2018.06.028 @ 2018. This manuscript version is made available under the CC-BY-NC-ND 4.0 license http://creativecommons.org/licenses/by-nc-nd/4.0/

\section{Appendix C.1. Small strains}

In the case of small strains, when $\|\mathbf{F}-\mathbf{I}\|<<1$, and assuming no dissipative processes in the bulk, the local dissipation per unit volume is due to the moving interface and reads $[16,48,51]$

$$
(\llbracket \psi \rrbracket-\langle\boldsymbol{\sigma}\rangle: \llbracket \varepsilon \rrbracket) \dot{\xi} \geq 0
$$

where the term in parenthesis is the thermodynamic driving force $\mathfrak{f}$ acting on the interface [48], and $\dot{\xi}$ is the rate of formation of new martensite volume fraction in the laminate. In (C.1), $\boldsymbol{\sigma}$ is the Cauchy stress tensor, $\boldsymbol{\varepsilon}=\operatorname{Sym}(\mathbf{F}-\mathbf{I})$ is the small strain tensor (Sym is the symmetrization operator), $\llbracket \cdot \rrbracket$ is the jump of a scalar or tensor quantity, $\langle\cdot\rangle$ the mean of a tensor quantity and $\psi$ is the Helmholtz free energy. The jump $\llbracket \cdot \rrbracket$ is defined as $\llbracket \cdot \rrbracket:=(\cdot)_{+}-(\cdot)_{-}$, and the mean as $2\langle\cdot\rangle:=(\cdot)_{+}+(\cdot)_{-}$. Positive quantities are on the side of the positive surface normal $\mathbf{n}^{(1)}$. The latter points also towards the positive direction of the interface velocity. Thus, the surface normal points in the austenite if the interface propagates in the austenite (fcc to bcc transformation), while it points in the martensite otherwise. It is also assumed, in case of forward (reverse) transformation, $\varepsilon_{A}=\varepsilon_{e, A}\left(\varepsilon_{A}=\varepsilon_{e, A}+\varepsilon_{\operatorname{tr}, A}\right)$ and $\varepsilon_{M}=\varepsilon_{e, M}+\varepsilon_{\operatorname{tr}, M}$ $\left(\varepsilon_{M}=\varepsilon_{e, M}\right)$.

We now develop Eq. (C.1) explicitly for the austenite to martensite transformation (developments for reverse martensite to austenite transformations are analogous). In the case of forward transformation, the first term in Eq. (C.1) reads

$$
\begin{aligned}
\llbracket \psi \rrbracket & =\psi_{A}-\psi_{M} \\
& =\Delta \psi_{A \rightarrow M}^{c}+\llbracket \psi^{e} \rrbracket
\end{aligned}
$$

where $\Delta \psi_{A \rightarrow M}^{c}$ is the difference in chemical free energy (per unit volume) between austenite and martensite $(\mathrm{A}=$ austenite, $\mathrm{M}=$ martensite). For example, in the atomistic simulations of Section $2, \Delta \psi_{A \rightarrow M}^{c}$ is the cohesive energy difference $\Delta E$ between fcc and bcc, per unit volume. This difference is positive if austenite is less stable than martensite (which favours transformation), and negative otherwise.

In Eq. (C.2), the elastic contribution to energy reads for small strains

$$
\psi_{I}^{e}=\frac{1}{2} \varepsilon_{e, I}: \mathbb{C}_{I}: \varepsilon_{e, I} \quad I=A, M
$$


This is a post-print of the following article: Maresca, F.; Kouznetsova, V.G.; Geers, M.G.D.; Curtin, W.A. Acta Materialia 2018,, 463-478. The formal publication is available at http://dx.doi.org/10.1016/j.actamat.2018.06.028 (C) 2018. This manuscript version is made available under the CC-BY-NC-ND 4.0 license http://creativecommons.org/licenses/by-nc-nd/4.0/

The second term in Eq. (C.1) yields

$$
\begin{aligned}
\langle\boldsymbol{\sigma}\rangle: \llbracket \varepsilon \rrbracket & =\frac{1}{2}\left(\boldsymbol{\sigma}_{A}+\boldsymbol{\sigma}_{M}\right):\left(\boldsymbol{\varepsilon}_{A}-\boldsymbol{\varepsilon}_{M}\right) \\
& =\frac{1}{2} \boldsymbol{\sigma}_{A}: \boldsymbol{\varepsilon}_{A}+\frac{1}{2} \boldsymbol{\sigma}_{M}: \boldsymbol{\varepsilon}_{A}-\frac{1}{2} \boldsymbol{\sigma}_{A}: \boldsymbol{\varepsilon}_{M}-\frac{1}{2} \boldsymbol{\sigma}_{M}: \boldsymbol{\varepsilon}_{M} \\
& =\frac{1}{2} \boldsymbol{\sigma}_{A}: \boldsymbol{\varepsilon}_{e, A}+\frac{1}{2} \boldsymbol{\sigma}_{M}: \boldsymbol{\varepsilon}_{e, A}-\frac{1}{2} \boldsymbol{\sigma}_{A}: \boldsymbol{\varepsilon}_{e, M}-\frac{1}{2} \boldsymbol{\sigma}_{A}: \boldsymbol{\varepsilon}_{t r, M}-\frac{1}{2} \boldsymbol{\sigma}_{M}: \boldsymbol{\varepsilon}_{e, M}-\frac{1}{2} \boldsymbol{\sigma}_{M}: \boldsymbol{\varepsilon}_{t r, M} \\
& =\psi_{A}^{e}-\psi_{M}^{e}-\frac{1}{2}\left(\boldsymbol{\sigma}_{A}+\boldsymbol{\sigma}_{M}\right): \boldsymbol{\varepsilon}_{t r, M}-\frac{1}{2} \varepsilon_{e, A}: \mathbb{C}_{A}: \boldsymbol{\varepsilon}_{e, M}-\frac{1}{2} \varepsilon_{e, M}: \mathbb{C}_{M}: \boldsymbol{\varepsilon}_{e, A} \\
& =\llbracket \psi^{e} \rrbracket-\frac{1}{2} \varepsilon_{e, A}: \llbracket \mathbb{C} \rrbracket: \varepsilon_{e, M}-\langle\boldsymbol{\sigma}\rangle: \boldsymbol{\varepsilon}_{t r, M} .
\end{aligned}
$$

Thus, the dissipation expressed by Eq. (C.1) reads

$$
\left(\Delta \psi_{A \rightarrow M}^{c}+\frac{1}{2} \varepsilon_{e, A}: \llbracket \mathbb{C} \rrbracket: \boldsymbol{\varepsilon}_{e, M}+\langle\boldsymbol{\sigma}\rangle: \boldsymbol{\varepsilon}_{t r, M}\right) \dot{\xi} \geq 0
$$

The left hand side of equation (C.5) is again the total driving force $\mathfrak{f}$ of the transformation. Note that in general $\left\|\varepsilon_{e}\right\|<<\left\|\varepsilon_{t r, M}\right\|$, and $\llbracket \mathbb{C} \rrbracket: \varepsilon_{e, M}<<\langle\boldsymbol{\sigma}\rangle$. Hence, the second term in parentheses of Eq. (C.5), which is the contribution of the elastic mismatch between the phases, can be neglected.

If the driving force in Eq. (C.5) is negative, the interface cannot propagate (i.e. $\dot{\xi}=0$ ). In case no stress is applied, transformation can occur only driven by a positive $\Delta \psi_{A \rightarrow M}^{c}$, namely when martensite is more stable than austenite. Thus the driving force for transformation reduces to

$$
\mathfrak{f}=\Delta \psi_{A \rightarrow M}^{c}+\langle\boldsymbol{\sigma}\rangle: \boldsymbol{\varepsilon}_{t r, M}
$$

Atomistic simulations show that phase transformation is achieved if the driving force reaches a threshold $\mathfrak{f}_{c}$. Therefore, like for plasticity, Kuhn-Tucker conditions can be defined as a function of a "transformation surface" $g=\mathfrak{f}-\mathfrak{f}_{c}$

$$
g=\mathfrak{f}-\mathfrak{f}_{c} \leq 0 \quad \dot{\xi} \geq 0 \quad g \dot{\xi}=0
$$

The connection with Eq. (3) is obtained by observing that, at the straight interface where compatibility and equilibrium conditions hold (Eqs. (8)), $\langle\boldsymbol{\sigma}\rangle: \boldsymbol{\varepsilon}_{t r, M}=m^{(1)} \boldsymbol{\sigma}:\left(\mathbf{s}^{(1)} \otimes \mathbf{n}^{(1)}\right)$ and hence $\bar{\varepsilon}_{t r} \tau=\langle\boldsymbol{\sigma}\rangle: \varepsilon_{t r, M}$, with $\tau$ the stress resolved on the transformation system and $m^{(1)}$ the magnitude of the transformation strain (which is a positive quantity). Therefore, the 
This is a post-print of the following article: Maresca, F.; Kouznetsova, V.G.; Geers, M.G.D.; Curtin, W.A. Acta Materialia 2018,, 463-478.. The formal publication is available at http://dx.doi.org/10.1016/j.actamat.2018.06.028 @ 2018. This manuscript version is made available under the CC-BY-NC-ND 4.0 license http://creativecommons.org/licenses/by-nc-nd/4.0/

Kuhn-Tucker conditions in Eq. (C.7) can be reformulated as a function of the surface $f=\tau-\tau_{c}$

$$
f=\tau-\tau_{c} \leq 0 \quad \dot{\varepsilon}_{\mathrm{tr}} \geq 0 \quad f \dot{\varepsilon}_{\mathrm{tr}}=0
$$

which is Equation (3).

By assuming like in Section 2, that forward transformation is controlled by the Peierls stress of fcc screws, and by noticing that the quantity $\tau m^{(1)}$ is the dissipated plastic work equal to the work performed to make the interface dislocation glide, we obtain as threshold for glide $\tau_{c}=\tau_{P, \text { fcc }}-\tau_{\Delta E}$, as adopted in the analysis of the critical stress for phase transformation, in Section 2 .

\section{Appendix C.2. Finite deformations}

In the finite deformation case, we assume for forward transformation $\mathbf{F}_{A}=\mathbf{F}_{e, A}$ and $\mathbf{F}_{M}=$ $\mathbf{F}_{e, M} \cdot \mathbf{F}_{t r, M}$, with $\mathbf{F}_{t r, M}$ a constant tensor defined by the crystallographic theory of martensite [12] and hence $\dot{\mathbf{F}}_{t r, M}=0$. Therefore, $\mathbf{P}_{M}: \dot{\mathbf{F}}_{M}=\mathbf{P}_{M}:\left(\dot{\mathbf{F}}_{e, M} \cdot \mathbf{F}_{t r, M}\right)=\left(\mathbf{P}_{M} \cdot \mathbf{F}_{t r, M}^{T}\right): \dot{\mathbf{F}}_{e, M}$, and any dislocation plasticity in the bulk phases is neglected. Note, that in this model of a moving interface, the deformation due to transformation characterizes the deformation of the new-formed martensite itself, and is not treated as an apparent plastic deformation as done in Section 3 of the present work (in which the martensite volume fraction is not updated, unlike e.g. [16]). Furthermore, we define the phase free energy as

$$
\begin{aligned}
\psi_{I} & =\psi_{I}^{c}+\psi_{I}^{e} \\
& =\psi_{I}^{c}+\frac{1}{2} \mathbf{E}_{e, I}: \mathbb{C}_{I}: \mathbf{E}_{e, I} \quad I=A, M .
\end{aligned}
$$

where $\mathbf{E}_{e, I}=\frac{1}{2}\left(\mathbf{C}_{e, I}-\mathbf{I}\right)$ is the Green-Lagrange elastic strain and $\mathbf{C}_{e, I}=\mathbf{F}_{e, I}^{T} \cdot \mathbf{F}_{e, I}$ is the right Cauchy-Green strain tensor.

In finite deformations, the local dissipation per unit volume due to the moving interface is [16]

$$
(\llbracket \psi \rrbracket-\langle\mathbf{P}\rangle: \llbracket \mathbf{F} \rrbracket) \dot{\xi} \geq 0
$$

In Equation (C.9)

$$
\begin{aligned}
\llbracket \psi \rrbracket & =\psi_{A}-\psi_{M} \\
& =\Delta \psi_{A \rightarrow M}^{c}+\llbracket \psi^{e} \rrbracket
\end{aligned}
$$


This is a post-print of the following article: Maresca, F.; Kouznetsova, V.G.; Geers, M.G.D.; Curtin, W.A. Acta Materialia 2018,, 463-478. The formal publication is available at http://dx.doi.org/10.1016/j.actamat.2018.06.028 @ 2018. This manuscript version is made available under the CC-BY-NC-ND 4.0 license http://creativecommons.org/licenses/by-nc-nd/4.0/

where

$$
\begin{aligned}
\llbracket \psi^{e} \rrbracket & =\psi_{A}^{e}-\psi_{M}^{e} \\
& =\frac{1}{2} \mathbf{E}_{e, A}: \mathbb{C}_{A}: \mathbf{E}_{e, A}-\frac{1}{2} \mathbf{E}_{e, M}: \mathbb{C}_{M}: \mathbf{E}_{e, M} \\
& =\frac{1}{4} \mathbf{C}_{e, A}: \overline{\mathbf{S}}_{A}-\frac{1}{4} \mathbf{C}_{e, M}: \overline{\mathbf{S}}_{M}-\frac{1}{4} \operatorname{tr}\left(\overline{\mathbf{S}}_{A}\right)+\frac{1}{4} \operatorname{tr}\left(\overline{\mathbf{S}}_{M}\right)
\end{aligned}
$$

while

$$
\begin{aligned}
\langle\mathbf{P}\rangle: \llbracket \mathbf{F} \rrbracket & =\frac{1}{2}\left(\mathbf{P}_{A}+\mathbf{P}_{M}\right):\left(\mathbf{F}_{A}-\mathbf{F}_{M}\right) \\
& =\frac{1}{2} \mathbf{P}_{A}: \mathbf{F}_{A}+\frac{1}{2} \mathbf{P}_{M}: \mathbf{F}_{A}-\frac{1}{2} \mathbf{P}_{A}: \mathbf{F}_{M}-\frac{1}{2} \mathbf{P}_{M}: \mathbf{F}_{M} \\
& =\frac{1}{2} \mathbf{P}_{A}: \mathbf{F}_{e, A}+\frac{1}{2} \mathbf{P}_{M}: \mathbf{F}_{e, A}-\frac{1}{2} \mathbf{P}_{A}: \mathbf{F}_{M}-\frac{1}{2} \mathbf{P}_{M}: \mathbf{F}_{M} \\
& =\frac{1}{2} \mathbf{C}_{e, A}: \overline{\mathbf{S}}_{A}-\frac{1}{2} \mathbf{C}_{e, M}: \overline{\mathbf{S}}_{M}+\frac{1}{2}\left(\mathbf{F}_{e, M}^{T} \cdot \mathbf{F}_{e, A} \cdot \mathbf{F}_{t r, M}^{-1}\right): \overline{\mathbf{S}}_{M}-\frac{1}{2}\left(\mathbf{F}_{e, A}^{T} \cdot \mathbf{F}_{M}\right): \overline{\mathbf{S}}_{A}
\end{aligned}
$$

Therefore, the term in parenthesis of Eq. (C.9) becomes

$$
\begin{aligned}
\llbracket \psi \rrbracket-\langle\mathbf{P}\rangle: \llbracket \mathbf{F} \rrbracket= & \Delta \psi_{A \rightarrow M}^{c}-\frac{1}{4} \mathbf{C}_{e, A}: \overline{\mathbf{S}}_{A}+\frac{1}{4} \mathbf{C}_{e, M}: \overline{\mathbf{S}}_{M}-\frac{1}{4} \operatorname{tr}\left(\overline{\mathbf{S}}_{A}\right)+\frac{1}{4} \operatorname{tr}\left(\overline{\mathbf{S}}_{M}\right) \\
& -\frac{1}{2}\left(\mathbf{F}_{e, M}^{T} \cdot \mathbf{F}_{e, A} \cdot \mathbf{F}_{t r, M}^{-1}\right): \overline{\mathbf{S}}_{M}+\frac{1}{2}\left(\mathbf{F}_{e, A}^{T} \cdot \mathbf{F}_{M}\right): \overline{\mathbf{S}}_{A}
\end{aligned}
$$

In the small elastic strain limit, which is the case for steels, $\mathbf{C}_{e, I} \simeq \mathbf{I}$. We can also consider $\mathbf{F}_{e, M}^{T} \cdot \mathbf{F}_{e, A} \simeq \mathbf{I}$ in small elastic strains, since for this system the components of deformation tangent to the interface normal $\mathbf{n}^{(1)}$ have to be equal, namely $\mathbf{F}_{e, M} \cdot\left(\mathbf{I}-\mathbf{n}^{(1)} \otimes \mathbf{n}^{(1)}\right)=\mathbf{F}_{e, A} \cdot(\mathbf{I}-$ $\left.\mathbf{n}^{(1)} \otimes \mathbf{n}^{(1)}\right)$, while the elastic components resolved on the interface relate to the interface stresses $\tau$, which are equal between austenite and martensite due to interface equilibrium conditions, and induce a shear strain difference $\frac{\tau}{\mu_{A}}-\frac{\tau}{\mu_{M}}$. Like for the case of small elastic strains, such shear shear strain difference can be neglected for small elastic phase mismatch. Furthermore, since $\mathbf{F}_{t r, M}=\mathbf{I}+m^{(1)} \mathbf{s}^{(1)} \otimes \mathbf{n}^{(1)}=\mathbf{I}+m^{(1)} \mathbf{N}_{t r, M}$, then $\mathbf{F}_{t r, M}^{-1} \simeq \mathbf{I}-m^{(1)} \mathbf{N}_{t r, M}$ due to the small non-shear components of $\mathbf{N}_{\mathrm{tr}, M}$; equality holds if $m^{(1)}$ is a pure shear. Therefore, in the small elastic strain limit and small phase mismatch Eq. (C.13) reads

$$
\llbracket \psi \rrbracket-\langle\mathbf{P}\rangle: \llbracket \mathbf{F} \rrbracket \simeq \Delta \psi_{A \rightarrow M}^{c}+m^{(1)}\langle\overline{\mathbf{S}}\rangle: \mathbf{N}_{t r, M} .
$$

This implies for small elastic strains, in case of straight interface and $\xi$ martensite volume fraction, the local dissipation

$$
\begin{aligned}
\mathcal{D} & =\dot{\xi} \Delta \psi_{A \rightarrow M}^{e}+\dot{\xi} m^{(1)}\langle\overline{\mathbf{S}}\rangle: \mathbf{N}_{t r, M} \\
& =\dot{\xi} \Delta \psi_{A \rightarrow M}^{e}+\langle\overline{\mathbf{S}}\rangle: \mathbf{L}_{t r, M} \geq 0,
\end{aligned}
$$


This is a post-print of the following article: Maresca, F.; Kouznetsova, V.G.; Geers, M.G.D.; Curtin, W.A. Acta Materialia 2018,, 463-478. The formal publication is available at http://dx.doi.org/10.1016/j.actamat.2018.06.028 @ 2018. This manuscript version is made available under the CC-BY-NC-ND 4.0 license http://creativecommons.org/licenses/by-nc-nd/4.0/

where $\mathbf{L}_{t r, M}=\dot{\xi} m^{(1)} \mathbf{N}_{t r, M}$ is the velocity gradient related to phase transformation.

From Eq. (C.15), it turns out that the driving force for transformation reads:

$$
\mathfrak{f}=\Delta \psi_{A \rightarrow M}^{c}+m^{(1)}\langle\overline{\mathbf{S}}\rangle: \mathbf{N}_{t r, M}
$$

By noticing that $m^{(1)}\langle\overline{\mathbf{S}}\rangle: \mathbf{N}_{t r, M}=m^{(1)} \tau$, the same analysis as in the small strain case yields to Equation (3).

\section{Appendix D. Transformation systems for each crystallographic variant}

Table D.2 lists all possible crystallographic variants for fcc/bcc transformation, in case of KS, NW and the in-situ transformation strain for the case $\phi=2.5^{\circ}$, with fcc/bcc lattice parameter ratio of $\mathrm{FeC}$ alloys [12]. 
This is a post-print of the following article: Maresca, F.; Kouznetsova, V.G.; Geers, M.G.D.; Curtin, W.A. Acta Materialia 2018,, 463-478. The formal publication is available at http://dx.doi.org/10.1016/j.actamat.2018.06.028 @ 2018. This manuscript version is made available under the CC-BY-NC-ND 4.0 license http://creativecommons.org/licenses/by-nc-nd/4.0/
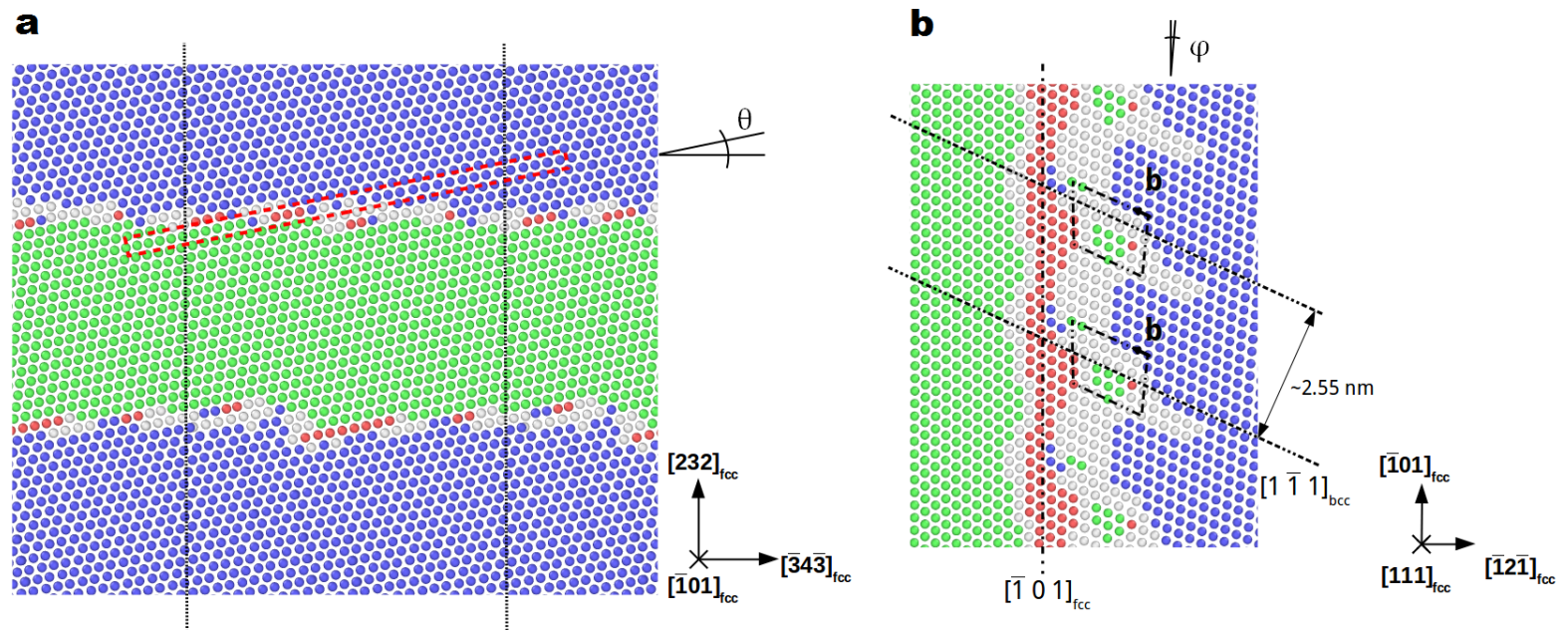

Figure 1: Interface defect structure of the bcc-fcc bicrystal. a, view from $[\overline{1} 01]_{\mathrm{fcc}}$, showing interface steps due to the misorientation $\theta$. Dashed black lines indicate the simulation cell boundaries. The red rectangle indicates the portion of $(111)_{\mathrm{fcc}} \|(011)_{\mathrm{bcc}}$ plane visualized in Figure $1 \mathrm{~b} . \mathbf{b}, \frac{a_{\mathrm{fcc}}}{2}[\overline{1} 01]$ screws and $\frac{a_{\mathrm{bcc}}}{2}[1 \overline{1} 1]$ screws with edge-character kinks viewed on a typical (111) fcc ledge. Crystallographic visualizations use OVITO [19] and adaptive Common Neighbor Analysis (CNA) [20], to label atoms as fcc (green), bcc (blue), hcp (red) and other (grey) according to local atomic environments. 
This is a post-print of the following article: Maresca, F.; Kouznetsova, V.G.; Geers, M.G.D.; Curtin, W.A. Acta Materialia 2018,, 463-478. The formal publication is available at http://dx.doi.org/10.1016/j.actamat.2018.06.028 @ 2018. This manuscript version is made available under the CC-BY-NC-ND 4.0 license http://creativecommons.org/licenses/by-nc-nd/4.0/

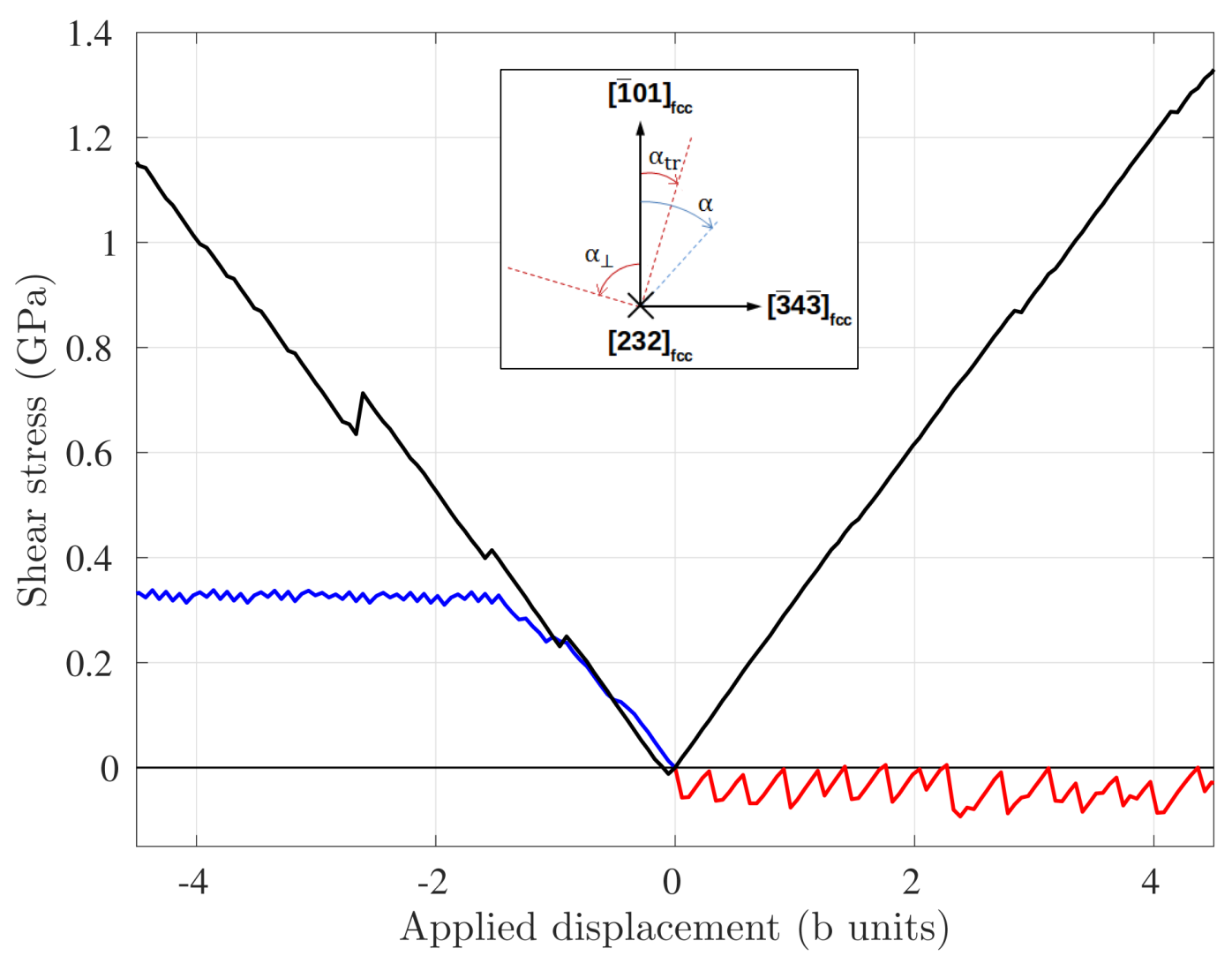

Figure 2: Orientation dependence of the stress-displacement response of the bicrystal. The resolved shear stress $\tau$ is calculated according to Eq. (1) and the displacement, in fcc Burgers vector units, is applied along $\alpha_{\mathrm{tr}}=19.5^{\circ}$ (blue and red curves) and $\alpha_{\perp}=-70.5^{\circ}$ (black curve). Inset: sketch of the load directions considered in simulations. The in-plane shear deformation directions are identified by an angle $\alpha \in\left[-180^{\circ}, 180^{\circ}\right]$. Positive $\alpha$ indicates an angle on the $(232)_{\text {fcc }}$ interface plane, starting from $[\overline{1} 01]_{\text {fcc }}$ along the clockwise direction. $\alpha_{\mathrm{tr}}=19.5^{\circ}$ is the phase transformation direction. 
This is a post-print of the following article: Maresca, F.; Kouznetsova, V.G.; Geers, M.G.D.; Curtin, W.A. Acta Materialia 2018,, 463-478. The formal publication is available at http://dx.doi.org/10.1016/j.actamat.2018.06.028 @ 2018. This manuscript version is made available under the CC-BY-NC-ND 4.0 license http://creativecommons.org/licenses/by-nc-nd/4.0/

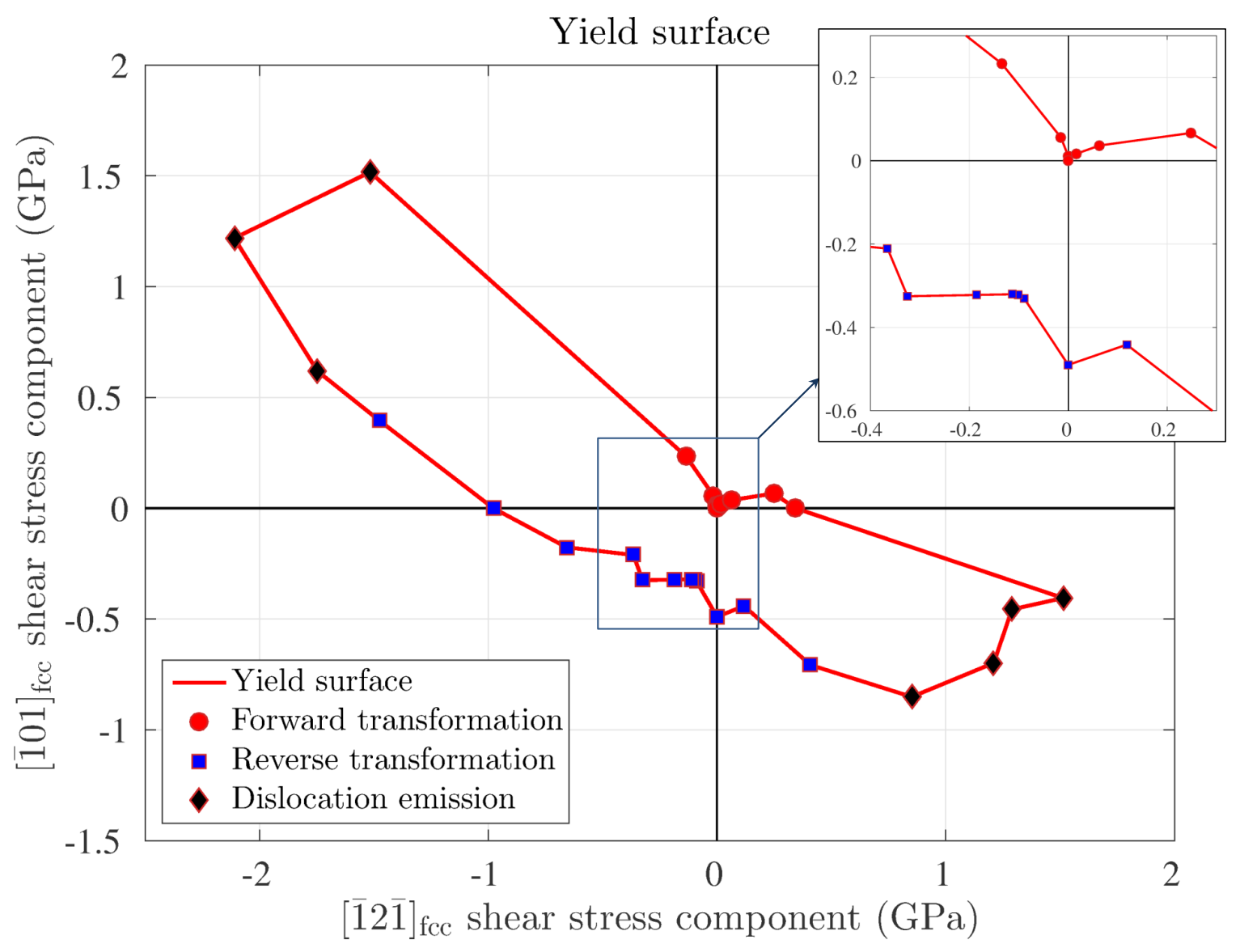

Figure 3: Yield surface of the fcc-bcc bicrystal for in-plane shear at $\mathbf{T}=\mathbf{0 K}$. The yield surface is constructed by connecting points in the stress diagram where at least $1 \mathrm{~b}$ displacement is accommodated by plastic phenomena: dislocation/twinning (stacking fault) emission, forward and reverse transformation. A zoom of the central part of the diagram is also given. 
This is a post-print of the following article: Maresca, F.; Kouznetsova, V.G.; Geers, M.G.D.; Curtin, W.A. Acta Materialia 2018,, 463-478. The formal publication is available at http://dx.doi.org/10.1016/j.actamat.2018.06.028 @ 2018. This manuscript version is made available under the CC-BY-NC-ND 4.0 license http://creativecommons.org/licenses/by-nc-nd/4.0/

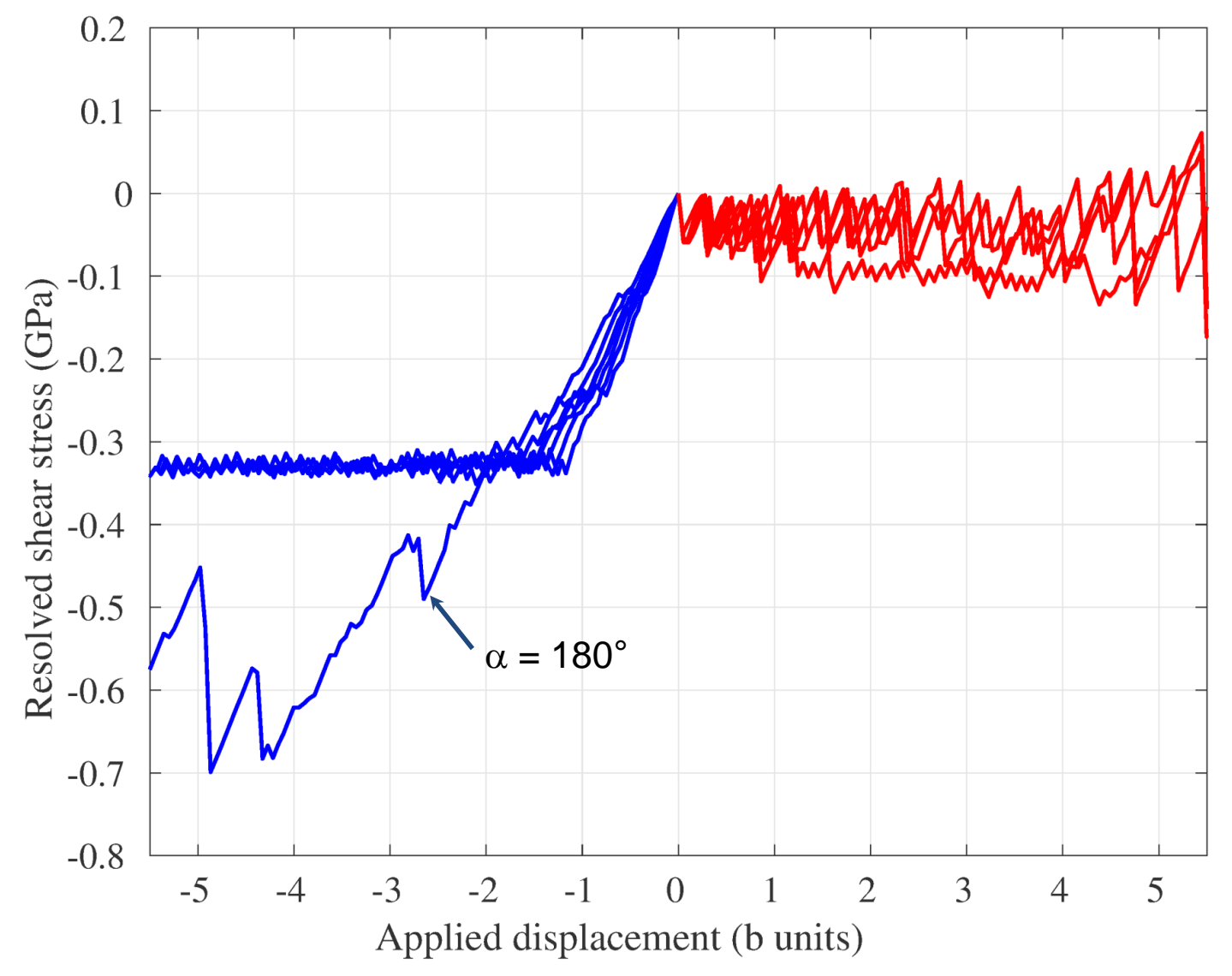

Figure 4: Resolved shear stress along the transformation direction $\alpha_{\mathrm{tr}}$. Stress versus applied displacement, resolved along the transformation direction $\mathbf{s}^{(1)}$, for all loading directions $\alpha$ where phase transformation occurs. Note that the case of reverse transformation $\alpha=180^{\circ}$ does not follow the general trend. 
This is a post-print of the following article: Maresca, F.; Kouznetsova, V.G.; Geers, M.G.D.; Curtin, W.A. Acta Materialia 2018,, 463-478.. The formal publication is available at http://dx.doi.org/10.1016/j.actamat.2018.06.028 @ 2018. This manuscript version is made available under the CC-BY-NC-ND 4.0 license http://creativecommons.org/licenses/by-nc-nd/4.0/

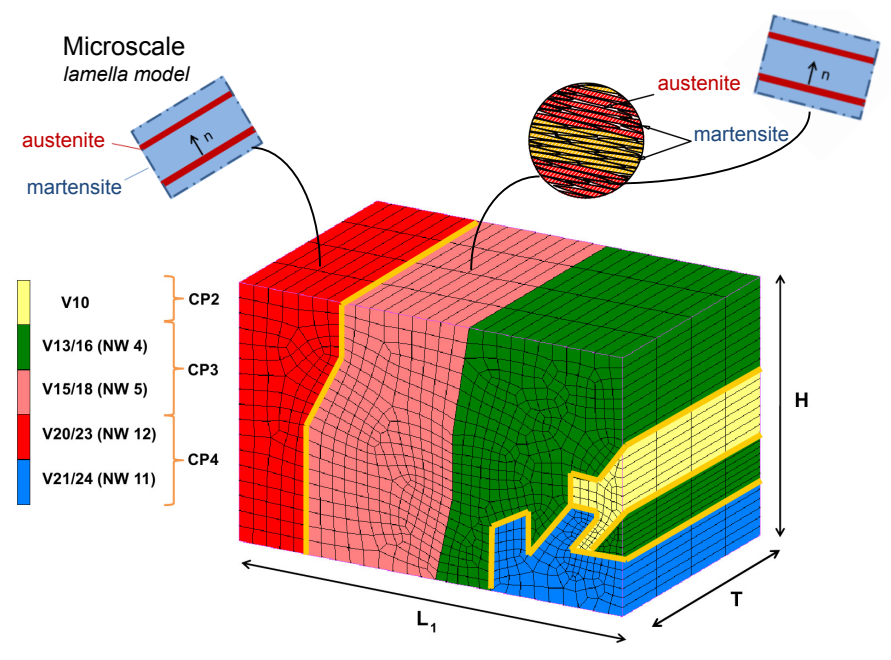

(a)

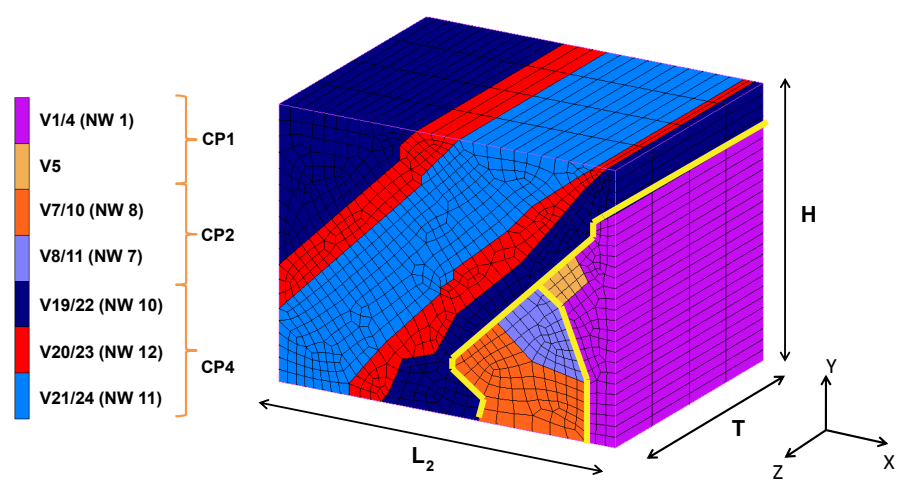

(b)

Figure 5: Model geometry and finite element mesh for the microstructure specimens studied here. (a) MP1 and (b) MP2, with crystallographic variants (V) (cf. Appendix D) and crystallographic packets (CP) indicated. The boundaries between variants belonging to different CP are highlighted by thick yellow lines.

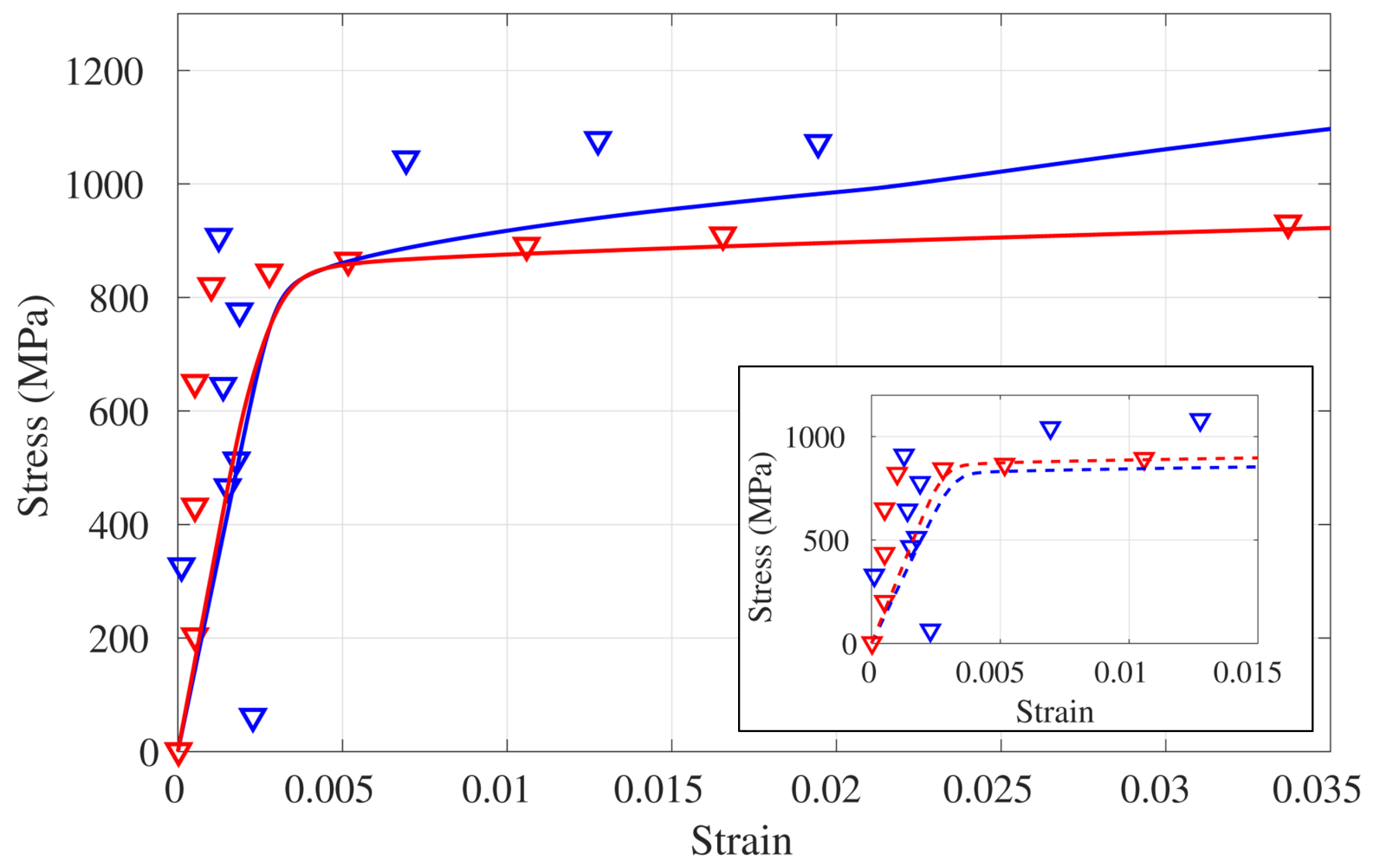

Figure 6: Experimental vs numerical flow curves. Simulated (solid lines) and experimental (symbols) stress-strain curves for microstructures MP1 (blue) and MP2 (red), where the flow parameters in the theory are identified from the experiments on microstructure MP2. Inset: predictions using only bcc plasticity with no fcc phase, where bcc flow parameters are identified from experiments on MP2. 
This is a post-print of the following article: Maresca, F.; Kouznetsova, V.G.; Geers, M.G.D.; Curtin, W.A. Acta Materialia 2018,, 463-478. The formal publication is available at http://dx.doi.org/10.1016/j.actamat.2018.06.028 @ 2018. This manuscript version is made available under the CC-BY-NC-ND 4.0 license http://creativecommons.org/licenses/by-nc-nd/4.0/

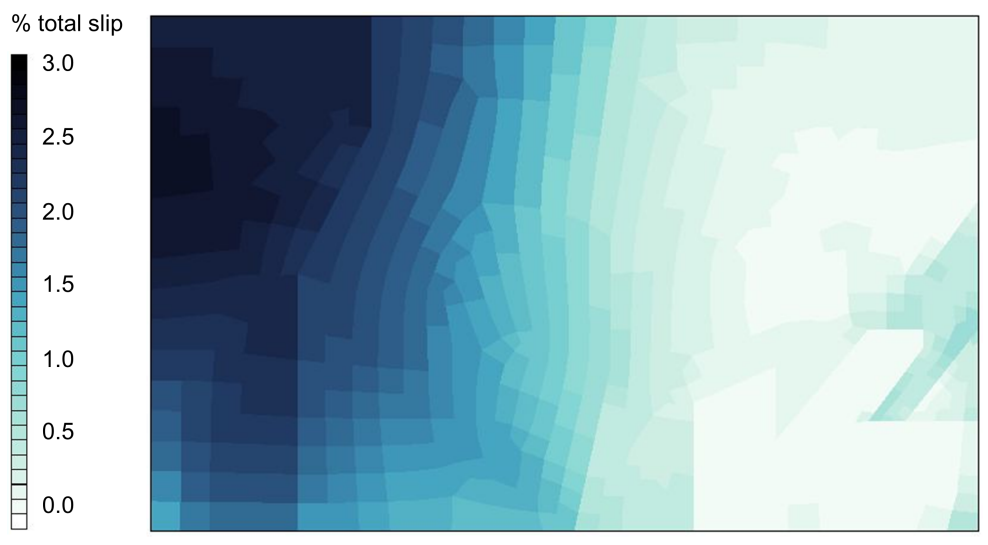

(a)

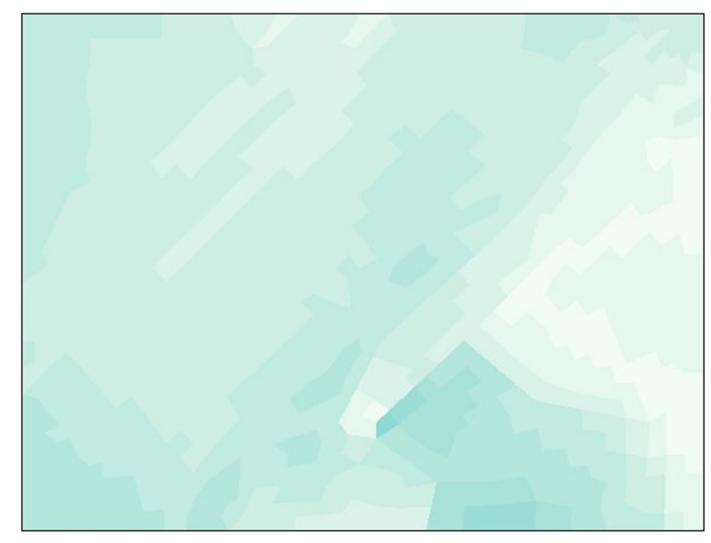

(b)

Figure 7: Simulated slip activity in the bcc laths. Total slip activity (sum of the slip on all active slip systems) in the bcc laths at $1 \%$ global tensile strain for (a) MP1 and (b) MP2 microstructures.

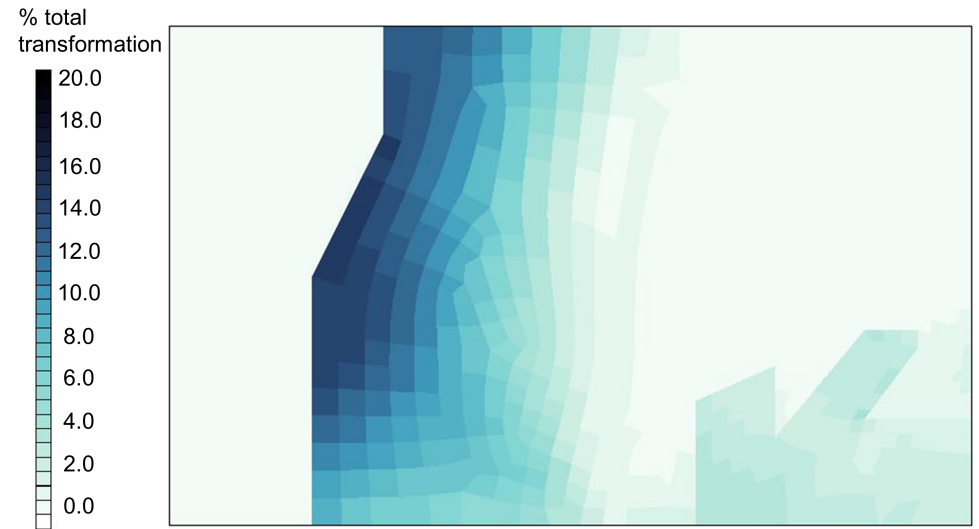

(a)

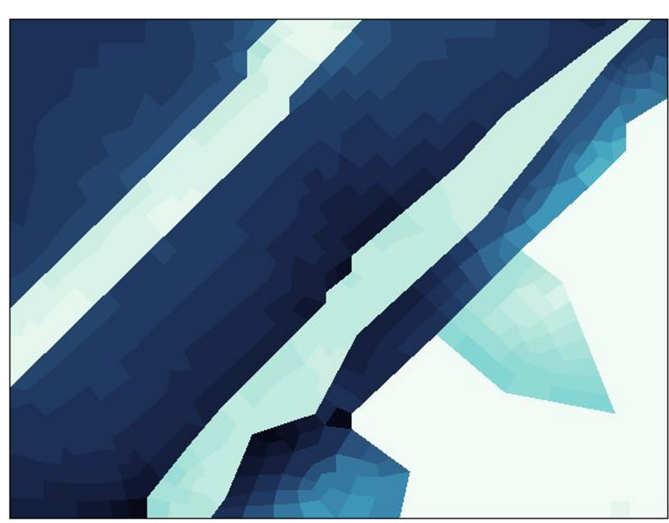

(b)

Figure 8: Simulated transformation strain activity. The apparent plasticity due to the transformation strain is shown, at $1 \%$ global uniaxial tensile strain, in (a) MP1 and (b) MP2 microstructures. Note the factor of nearly 7 difference in the scale compared to Figure 7 . 
This is a post-print of the following article: Maresca, F.; Kouznetsova, V.G.; Geers, M.G.D.; Curtin, W.A. Acta Materialia 2018,, 463-478. The formal publication is available at http://dx.doi.org/10.1016/j.actamat.2018.06.028 @ 2018. This manuscript version is made available under the CC-BY-NC-ND 4.0 license http://creativecommons.org/licenses/by-nc-nd/4.0/

\section{a}

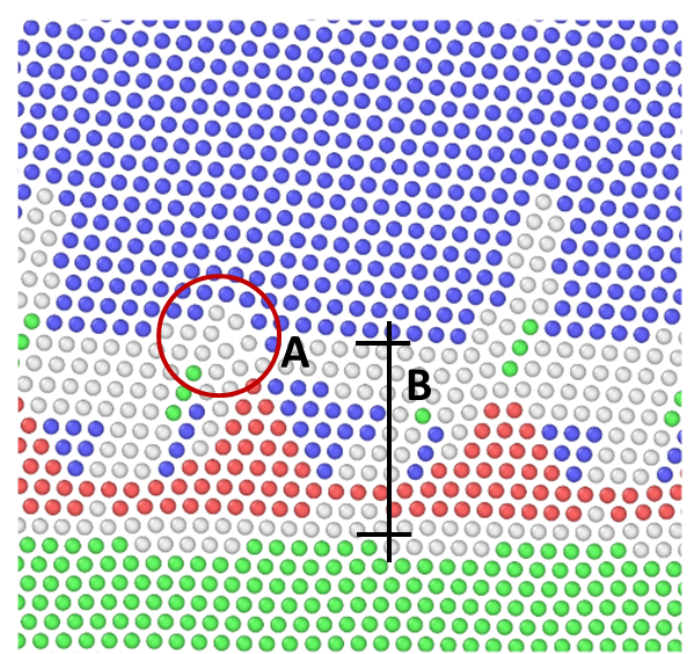

\section{b}

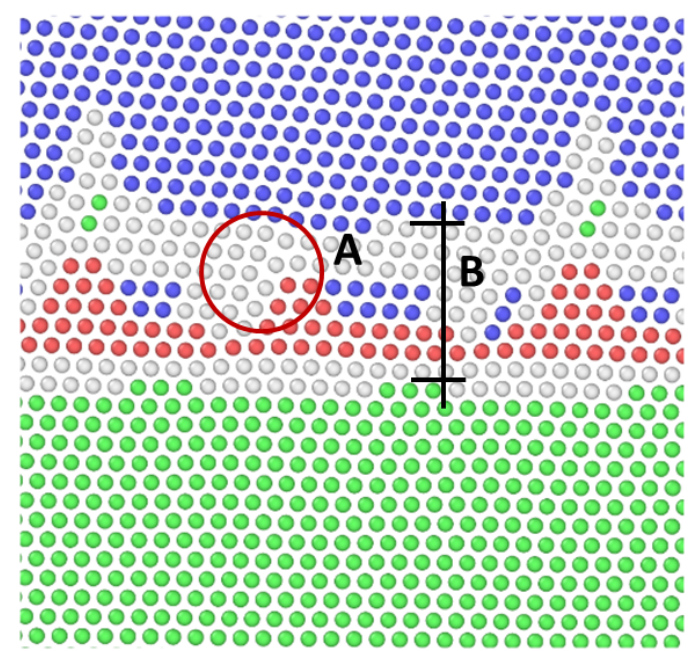

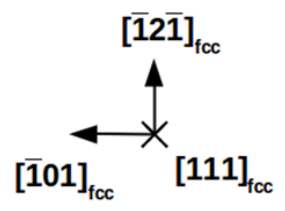

Figure B.9: Interface rearrangements during bcc to fcc reverse transformation. a, (111) fcc interface prior to deformation. $\mathbf{b}$, interface after reverse transformation is activated. Local atomic rearrangements occur before the transformation can take place, indicated by labels A and B. A refers to the rearrangements of the $[1 \overline{1} 1]_{\text {bcc }}$ bcc edge-character kink cores, while $\mathrm{B}$ indicates the shrinking of the interface steps from $\sim 2 \mathrm{~nm}$ to $\sim 1.6 \mathrm{~nm}$. Colors show local atomic environments according to CNA. 
This is a post-print of the following article: Maresca, F.; Kouznetsova, V.G.; Geers, M.G.D.; Curtin, W.A. Acta Materialia 2018,, 463-478. The formal publication is available at http://dx.doi.org/10.1016/j.actamat.2018.06.028 @ 2018. This manuscript version is made available under the CC-BY-NC-ND 4.0 license http://creativecommons.org/licenses/by-nc-nd/4.0/

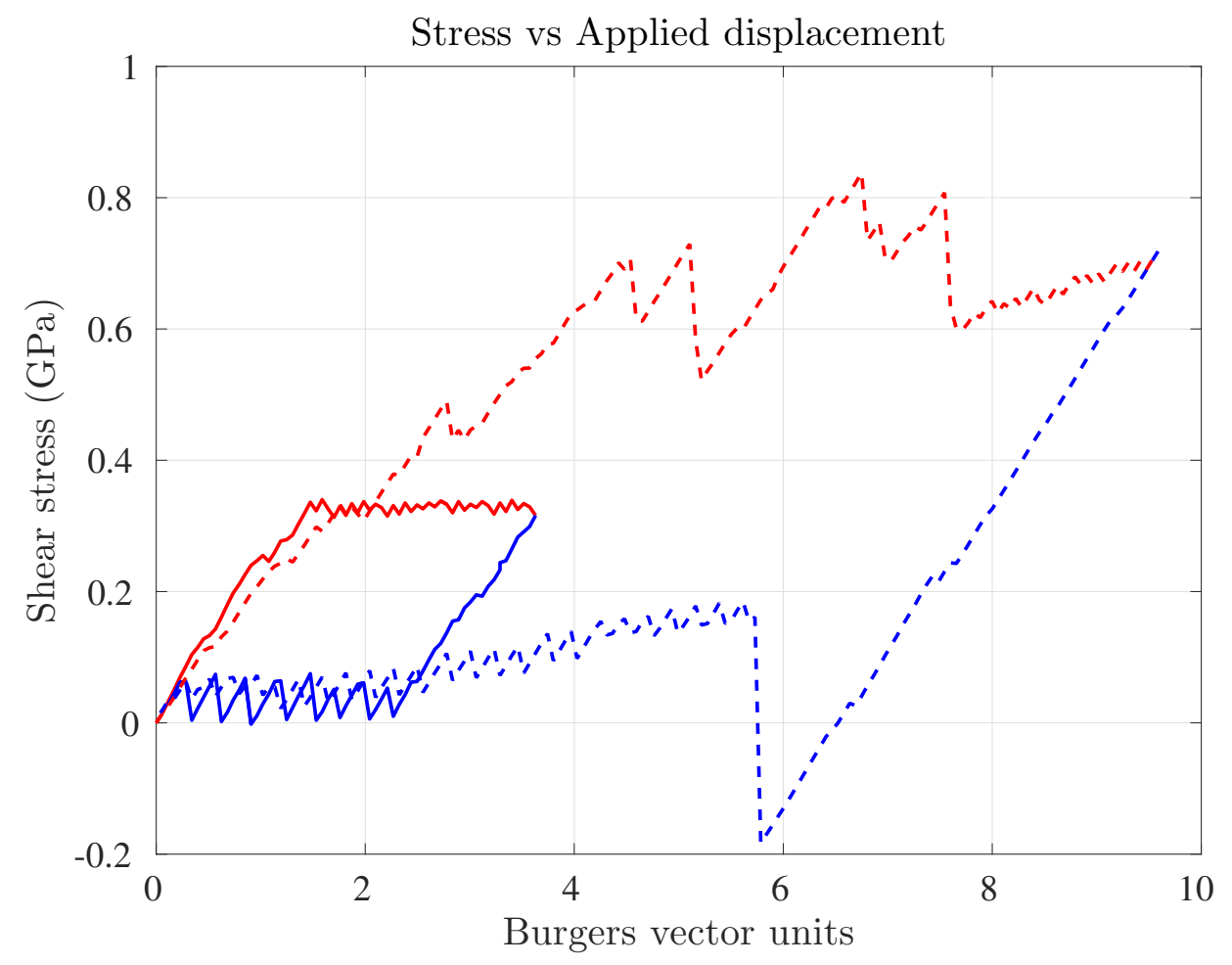

Figure B.10: Cyclic loading of the bcc-fcc interface. Solid lines refer to a load along $\alpha_{\mathrm{tr}}=19.5^{\circ}$, first applied in the reverse sense (red) and later on in the forward sense (blue), after some bcc to fcc transformation has developed. Dashed lines refer to a load along $\alpha=0^{\circ}$ (reverse in red, forward in blue). This second case also delivers reverse transformation, but at much higher stresses than $\alpha_{\text {tr }}$ due to the bcc kinks crossing the interface steps from bcc to fcc. 
This is a post-print of the following article: Maresca, F.; Kouznetsova, V.G.; Geers, M.G.D.; Curtin, W.A. Acta Materialia 2018,, 463-478. The formal publication is available at http://dx.doi.org/10.1016/j.actamat.2018.06.028 @ 2018. This manuscript version is made available under the CC-BY-NC-ND 4.0 license http://creativecommons.org/licenses/by-nc-nd/4.0/

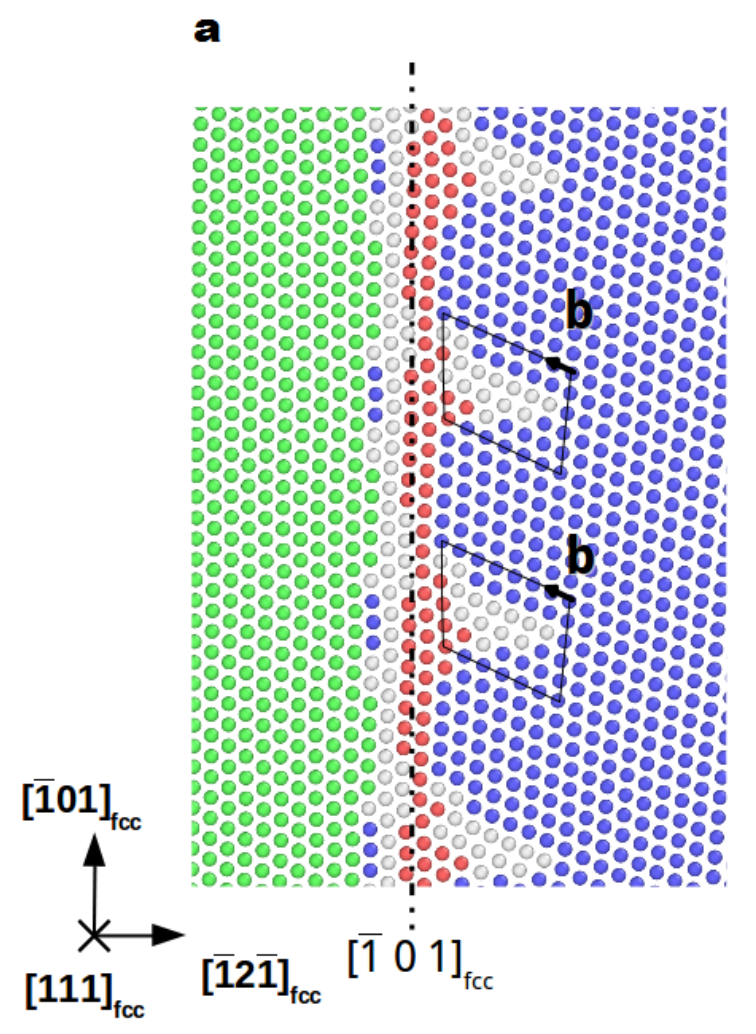

b

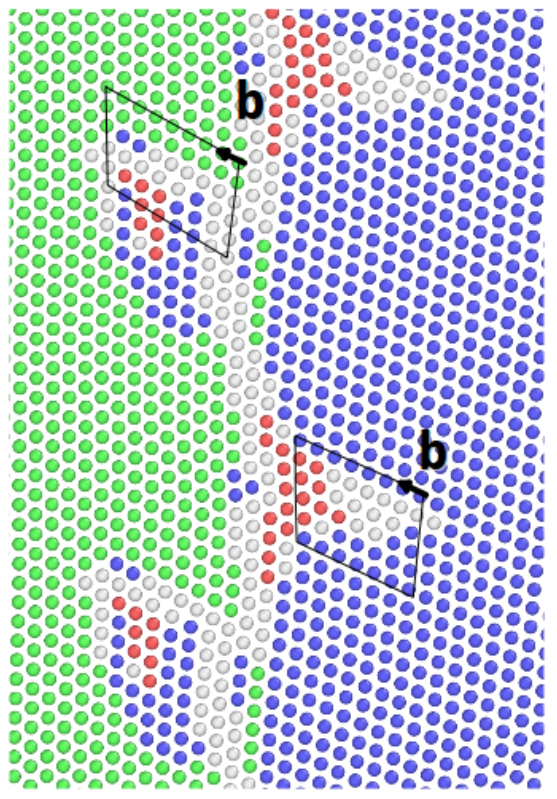

Figure B.11: Edge defects crossing the interface. a, interface structure before loading and $\mathbf{b}$, interface structure after loading at $\alpha=180^{\circ}$. One of the two bcc kinks in the periodic simulation cell has crossed the interface screw during the reverse transformation. Colors show local atomic environments according to CNA. 
This is a post-print of the following article: Maresca, F.; Kouznetsova, V.G.; Geers, M.G.D.; Curtin, W.A. Acta Materialia 2018,, 463-478.. The formal publication is available at http://dx.doi.org/10.1016/j.actamat.2018.06.028 @ 2018. This manuscript version is made available under the CC-BY-NC-ND 4.0 license http://creativecommons.org/licenses/by-nc-nd/4.0/

Table D.2: Crystallographic variants classified with respect to KS (label numbering based on [27]). NW variants (and fcc directions $\|[100]_{\alpha^{\prime}}$ )are obtained by clockwise $(+)$ or counter-clockwise $(-)$ rotations $\varphi_{N W}= \pm 5.26^{\circ}$ with respect to the positive KS crystallographic plane axis. All variants are classified by crystallographic packets (see [27]) and Bain Groups [54]. Habit planes and transformation directions are provided for the variants considered for modeling $\mathrm{FeC}$, characterized by $\varphi= \pm 2.5^{\circ}$ (same sign as related $\mathrm{NW}$ variant) and $a_{\mathrm{fcc}} / a_{\mathrm{bcc}}=1.274$. For this specific orientation and lattice parameter ratio, $h=2.0, k=2.2054$ and $l=3.0125$ for the habit planes, while $a=0.1614, b=0.5673$ and $c=0.8075$ for the transformation direction.

\begin{tabular}{|c|c|c|c|c|c|c|c|}
\hline $\mathrm{KS}$ & \| planes & \| directions & Bain Group & NW & NW dir. & Habit pl. & Transf. dir \\
\hline $\mathrm{V} 1$ & & {$[\overline{1} 01]_{\gamma} \|[\overline{1} \overline{1} 1]_{\alpha^{\prime}}$} & BG1 & NW1(+) & {$[1 \overline{1} 0]_{\gamma}$} & $(k l h)_{\gamma}$ & {$[\bar{c} a b]_{\gamma}$} \\
\hline $\mathrm{V} 2$ & & {$[10 \overline{1}]_{\gamma} \|[1 \overline{1} 1]_{\alpha^{\prime}}$} & BG2 & NW3(-) & {$[01 \overline{1}]_{\gamma}$} & $(\bar{h} \overline{l k})_{\gamma}$ & {$[\bar{b} \bar{a} c]_{\gamma}$} \\
\hline $\mathrm{V} 3$ & $(111)_{\gamma} \|$ & {$[01 \overline{1}]_{\gamma} \|[\overline{1} \overline{1} 1]_{\alpha^{\prime}}$} & BG3 & NW2(+) & {$[\overline{1} 01]_{\gamma}$} & $(\overline{l h} \bar{k})_{\gamma}$ & {$[\bar{a} \bar{b} c]_{\gamma}$} \\
\hline $\mathrm{V} 4$ & $(011)_{\alpha^{\prime}}$ & {$[0 \overline{1} 1]_{\gamma} \|[1 \overline{1} 1]_{\alpha^{\prime}}$} & BG1 & NW1(-) & {$[1 \overline{1} 0]_{\gamma}$} & $(l k h)_{\gamma}$ & {$[a \bar{c} b]_{\gamma}$} \\
\hline $\mathrm{V} 5$ & & {$[1 \overline{1} 0]_{\gamma} \|[\overline{1} \overline{1} 1]_{\alpha^{\prime}}$} & $\mathrm{BG} 2$ & NW3(+) & {$[01 \overline{1}]_{\gamma}$} & $(h k l)_{\gamma}$ & {$[b \bar{c} a]_{\gamma}$} \\
\hline V6 & & {$[\overline{1} 10]_{\gamma} \|[1 \overline{1} 1]_{\alpha^{\prime}}$} & BG3 & NW2(-) & {$[\overline{1} 01]_{\gamma}$} & $(k h l)_{\gamma}$ & {$[\bar{c} b a]_{\gamma}$} \\
\hline $\mathrm{V} 7$ & & {$[10 \overline{1}]_{\gamma} \|[\overline{1} \overline{1} 1]_{\alpha^{\prime}}$} & BG2 & NW8(-) & {$[011]_{\gamma}$} & $(\bar{h} l \bar{k})_{\gamma}$ & {$[\bar{c} \bar{a} b]_{\gamma}$} \\
\hline $\mathrm{V} 8$ & & {$[\overline{1} 01]_{\gamma} \|[1 \overline{1} 1]_{\alpha^{\prime}}$} & BG1 & NW7(+) & {$[\overline{1} \overline{1} 0]_{\gamma}$} & $(k \bar{l} h)_{\gamma}$ & {$[\bar{b} a c]_{\gamma}$} \\
\hline V9 & $(1 \overline{1} 1)_{\gamma} \|$ & {$[\overline{1} \overline{1} 0]_{\gamma} \|[\overline{1} \overline{1} 1]_{\alpha^{\prime}}$} & BG3 & NW9(-) & {$[10 \overline{1}]_{\gamma}$} & $(k \bar{h} l)_{\gamma}$ & {$[\bar{a} b c]_{\gamma}$} \\
\hline V10 & $(011)_{\alpha^{\prime}}$ & {$[110]_{\gamma} \|[1 \overline{1} 1]_{\alpha^{\prime}}$} & BG2 & NW8(+) & {$[011]_{\gamma}$} & $(h \bar{k} l)_{\gamma}$ & {$[a c b]_{\gamma}$} \\
\hline V11 & & {$[011]_{\gamma} \|[\overline{1} \overline{1} 1]_{\alpha^{\prime}}$} & BG1 & NW7(-) & {$[\overline{1} \overline{1} 0]_{\gamma}$} & $(l \bar{k} h)_{\gamma}$ & {$[b c a]_{\gamma}$} \\
\hline V12 & & {$[0 \overline{1} \overline{1}]_{\gamma} \|[1 \overline{1} 1]_{\alpha^{\prime}}$} & BG3 & NW9(+) & {$[10 \overline{1}]_{\gamma}$} & $(\bar{l} h \bar{k})_{\gamma}$ & {$[\bar{c} \bar{b} a]_{\gamma}$} \\
\hline V13 & & {$[0 \overline{1} 1]_{\gamma} \|[\overline{1} \overline{1} 1]_{\alpha^{\prime}}$} & BG1 & NW4(+) & {$[110]_{\gamma}$} & $(\bar{l} k h)_{\gamma}$ & {$[\bar{a} \bar{c} b]_{\gamma}$} \\
\hline V14 & & {$[01 \overline{1}]_{\gamma} \|[1 \overline{1} 1]_{\alpha^{\prime}}$} & BG3 & NW6(-) & {$[\overline{1} 0 \overline{1}]_{\gamma}$} & $(\bar{l} h k)_{\gamma}$ & {$[\bar{b} \bar{c} a]_{\gamma}$} \\
\hline V15 & $(\overline{1} 11)_{\gamma} \|$ & {$[\overline{1} 0 \overline{1}]_{\gamma} \|[\overline{1} \overline{1} 1]_{\alpha^{\prime}}$} & $\mathrm{BG} 2$ & NW5 $(+)$ & {$[0 \overline{1} 1]_{\gamma}$} & $(\bar{h} l k)_{\gamma}$ & {$[c b a]_{\gamma}$} \\
\hline V16 & $(011)_{\alpha^{\prime}}$ & {$[101]_{\gamma} \|[1 \overline{1} 1]_{\alpha^{\prime}}$} & BG1 & NW4(-) & {$[110]_{\gamma}$} & $(\bar{k} l h)_{\gamma}$ & {$[c a b]_{\gamma}$} \\
\hline V17 & & {$[110]_{\gamma} \|[\overline{1} \overline{1} 1]_{\alpha^{\prime}}$} & BG3 & NW6(+) & {$[\overline{1} 0 \overline{1}]_{\gamma}$} & $(\bar{k} h l)_{\gamma}$ & {$[\bar{b} a \bar{c}]_{\gamma}$} \\
\hline V18 & & {$[\overline{1} \overline{1} 0]_{\gamma} \|[1 \overline{1} 1]_{\alpha^{\prime}}$} & BG2 & NW5(-) & {$[0 \overline{1} 1]_{\gamma}$} & $(\bar{h} k l)_{\gamma}$ & {$[\bar{a} b \bar{c}]_{\gamma}$} \\
\hline V19 & & {$[\overline{1} 10]_{\gamma} \|[\overline{1} \overline{1} 1]_{\alpha^{\prime}}$} & BG3 & NW10(-) & {$[101]_{\gamma}$} & $(\bar{k} \bar{h} l)_{\gamma}$ & {$[c \bar{a} b]_{\gamma}$} \\
\hline V20 & & {$[1 \overline{1} 0]_{\gamma} \|[1 \overline{1} 1]_{\alpha^{\prime}}$} & BG2 & NW12(+) & {$[0 \overline{1} \overline{1}]_{\gamma}$} & $(\bar{h} \bar{k} l)_{\gamma}$ & {$[\bar{b} \bar{a} \bar{c}]_{\gamma}$} \\
\hline V21 & $(11 \overline{1})_{\gamma} \|$ & {$[0 \overline{1} \overline{1}]_{\gamma} \|[\overline{1} \overline{1} 1]_{\alpha^{\prime}}$} & BG1 & NW11(+) & {$[\overline{1} 10]_{\gamma}$} & $(\overline{l k} h)_{\gamma}$ & {$[c \bar{b} a]_{\gamma}$} \\
\hline $\mathrm{V} 22$ & $(011)_{\alpha^{\prime}}$ & {$[011]_{\gamma} \|[1 \overline{1} 1]_{\alpha^{\prime}}$} & BG3 & NW10(+) & {$[101]_{\gamma}$} & $(\overline{l h} k)_{\gamma}$ & {$[\bar{a} c b]_{\gamma}$} \\
\hline V23 & & {$[101]_{\gamma} \|[\overline{1} \overline{1} 1]_{\alpha^{\prime}}$} & BG2 & NW12(-) & {$[0 \overline{1} \overline{1}]_{\gamma}$} & $(\bar{h} \bar{l} k)_{\gamma}$ & {$[\bar{b} c a]_{\gamma}$} \\
\hline $\mathrm{V} 24$ & & {$[\overline{1} 0 \overline{1}]_{\gamma} \|[1 \overline{1} 1]_{\alpha^{\prime}}$} & BG1 & NW11(-) & {$[\overline{1} 10]_{\gamma}$} & $(\bar{k} \bar{l} h)_{\gamma}$ & {$[\bar{a} \bar{b} \bar{c}]_{\gamma}$} \\
\hline
\end{tabular}

